\title{
Aroylethenesulfonylacetic Acid Methyl Ester-A Synthon for Novel Sulfone Linked Bis Heterocycles
}

\author{
Venkatapuram Padmavathi, * Annaji Venkata Nagendra Mohan, Konda Mahesh, and \\ Adivireddy PADMAJA
}

Department of Chemistry, Sri Venkateswara University; Tirupati-517 502, India.

Received February 5, 2008; accepted March 22, 2008; published online March 27, 2008

\begin{abstract}
Novel sulfone linked bis heterocycles containing two different heterocyclic rings viz., pyrazoline in combination with thiadiazole, oxadiazole and triazole were synthesized and studied their antimicrobial activity.
\end{abstract}

Key words bis heterocycle; thiadiazole; oxadiazole; triazole; antimicrobial activity

Amongst different heterocyclic systems five membered heterocycles represent a class of compounds of biological significance. In fact, azoles occupy a unique place in the realm of natural and synthetic organic chemistry. These compounds have intrinsic biological activities and constitute the structural feature of many bioactive compounds. 1,3,4-Oxadiazoles exhibit a broad spectrum of biological activities such as anti human immunodeficiency virus (HIV), antibacterial and antifungal. ${ }^{1,2)}$ Besides, triazoles possess antifungal activity and reduce toxicity when compared with the imidazole antifungals. ${ }^{3-8)}$ Flucanazole and itraconazole are most frequently used antifungal drugs. 1,3,4-Thiadiazoles are associated with diverse biological activities probably due to toxophoric $-\mathrm{N}=\mathrm{C}-\mathrm{S}$ group. ${ }^{9-12)}$ In fact, the advent of sulfur drugs and the discovery of mesoionic compounds greatly accelerated the rate of progress in the field of thiadiazoles. 5Unsubstituted 1,3,4-thiadiazoles are used as intermediates in the synthesis of therapeutically potent antibiotic cefazolin. ${ }^{13)}$ In addition, pyrazolines have gained importance due to their various chemotherapeutic properties. Celecoxib, a pyrazole derivative is now widely used in the market as an anti-inflammatory drug. ${ }^{14)}$ Hence, it is considered worthwhile to prepare molecules having pyrazoline and oxadiazole/thiadiazole/ triazole rings.

Chemistry The general synthetic pathway discussed hereafter is depicted in Charts 1 and 2. The synthetic intermediate aroylethenesulfonylacetic acid (3) is prepared by the condensation of 1-aroyl-2-chloroethene (1) with mercaptoacetic acid in the presence of sodium hydroxide in methanol followed by oxidation with hydrogen peroxide in acetic acid. The ${ }^{1} \mathrm{H}$-NMR spectrum of 3a showed a singlet at $4.38 \mathrm{ppm}$ for methylene protons and two doublets at 7.66, $7.96 \mathrm{ppm}$ for olefinic protons. The downfield shift is assigned to the one adjacent to $\mathrm{SO}_{2}$ group. The methyl ester of $\mathbf{3}(\mathbf{4})$ is prepared by treating 3 with methanol in conc. $\mathrm{H}_{2} \mathrm{SO}_{4}$. When aroylethenesulfonylacetic acid methyl ester $\mathbf{4}$ is made to react with hydrazine hydrate instead of the expected acid hydrazide, a mixture of products are obtained. The two are separated by column chromatography and identified as 1,1dioxo-6-phenacyl-[1,4,5]thiadiazinan-3-one (5) and (3-aryl4,5-dihydro- $1 H$-pyrazole-5-sulfonyl)-acetic acid methyl ester (6) by spectral parameters. The ${ }^{1} \mathrm{H}-\mathrm{NMR}$ spectrum of $\mathbf{5 a}$ showed a singlet, a doublet and a multiplet at $4.28,3.50$ and 4.57-4.59 ppm for $\mathrm{H}-2, \mathrm{CO}-\mathrm{CH}_{2}$ and $\mathrm{H}-6$ respectively. Two broad singlets are observed at 2.82 and $8.50 \mathrm{ppm}$ due to $\mathrm{NH}$ which disappeared on deuteration. The downfield absorption is assigned to the one adjacent to $\mathrm{C}=\mathrm{O}$ group. However, the ${ }^{1} \mathrm{H}-\mathrm{NMR}$ spectrum of $\mathbf{6 a}$ displayed a singlet at $3.72 \mathrm{ppm}$ for methoxy protons of carbomethoxy group, another singlet at $4.39 \mathrm{ppm}$ for $\mathrm{SO}_{2}-\mathrm{CH}_{2}$, a multiplet at $4.62-4.65$ for $\mathrm{H}-5$ and a doublet at $3.53 \mathrm{ppm}$ for $\mathrm{H}-4$. In order to get the desired bis heterocycles, the olefinic moiety in $\mathbf{4}$ is used to develop pyrazoline ring by 1,3-dipolar cycloaddition of diazomethane. In 1,2-disubstituted ethylenes bearing two vicinal electron withdrawing substituents a regioisomeric mixture of cycloadducts are expected. However, the reaction of 1-aryl-2aroylethylenes with diazomethane and its derivatives produced exclusively 3-aroyl-4-aryl-2-pyrazolines. ${ }^{15)}$ Similarly, the cycloaddition of diazomethane to 1-aroyl-2-styrylsulfonylethenes gave 3-aroyl-4-styrylsulfonyl-2-pyrazolines. ${ }^{16}$ ) The treatment of $\mathbf{4}$ with diazomethane in the presence of $\mathrm{Et}_{3} \mathrm{~N}$ at $-20^{\circ} \mathrm{C}$ for $48 \mathrm{~h}$ gave a solid which is identified as (3-aroyl-4,5-dihydro- $1 H$-pyrazole-4-sulfonyl)-acetic acid methyl ester (7). The addition of diazomethane to 4 may produce regioisomers. However, we have isolated only one pure regioisomer. A small amount of the other isomer if any, formed could not be isolated by this process. The ${ }^{1} \mathrm{H}-\mathrm{NMR}$ spectrum of 7a shows an AMX splitting pattern for the pyrazoline ring protons thus exhibiting three double doublets at $5.07\left(\mathrm{H}_{\mathrm{A}}\right), 4.53\left(\mathrm{H}_{\mathrm{M}}\right)$ and $3.94 \mathrm{ppm}\left(\mathrm{H}_{\mathrm{X}}\right)$ respectively, in addition to the signals due to methylene and methoxy protons. The ${ }^{13} \mathrm{C}-\mathrm{NMR}$ spectrum of $\mathbf{7 a}$ displayed a signal at $185.6 \mathrm{ppm}$ due to carbonyl carbon, a characteristic signal for carbonyl carbon adjacent to an imine carbon. The articulation of oxadiazole, thiadiazole and triazole rings is made by the use of ester moiety. The compound 7 on reaction with hydrazine hydrate gave the corresponding acid hydrazide 8 . The

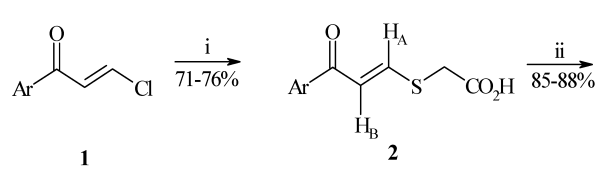<smiles>[13CH3]C([14CH3])=CS(=O)(=O)CC(=O)O</smiles>

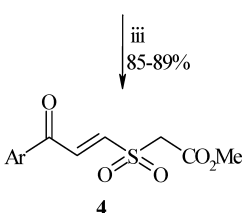

(i) $\mathrm{SHCH}_{2} \mathrm{CO}_{2} \mathrm{H} / \mathrm{NaOH} / \mathrm{MeOH}, 0{ }^{\circ} \mathrm{C}, 3-4 \mathrm{~h}$

(ii) $\mathrm{H}_{2} \mathrm{O}_{2} / \mathrm{AcOH}, 0^{\circ} \mathrm{C}, 4 \mathrm{~h}$

(iii) $\mathrm{H}_{2} \mathrm{SO}_{4} / \mathrm{MeOH}$, reflux, 5-7 h

a: $\mathrm{Ar}=\mathrm{Ph}$

b: $\mathrm{Ar}=4-\mathrm{Me} \cdot \mathrm{Ph}$

c: $\mathrm{Ar}=4-\mathrm{Cl} . \mathrm{Ph}$ 


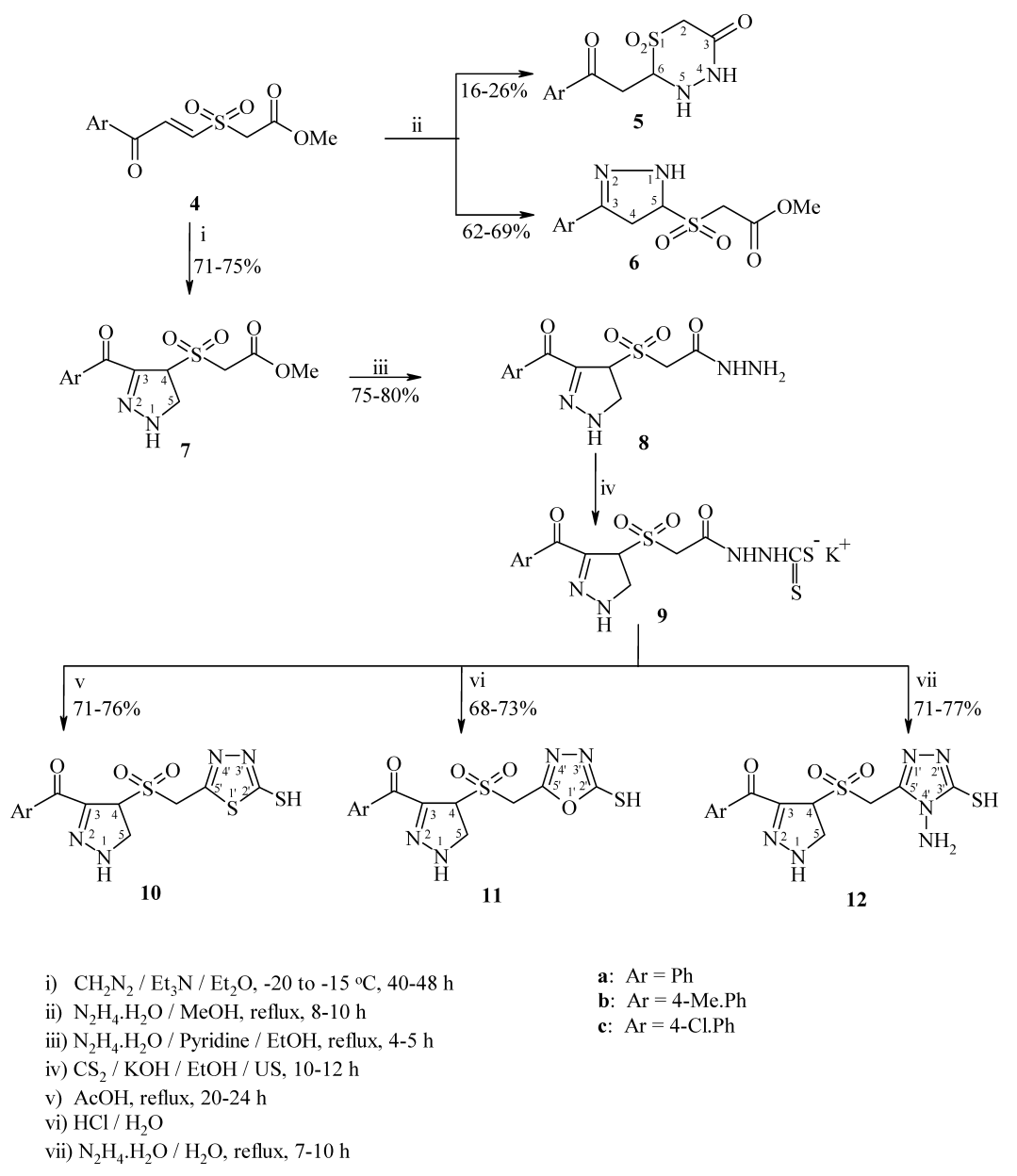

Chart 2

Table 1. The in Vitro Antibacterial Activity of 10-12

\begin{tabular}{|c|c|c|c|c|c|}
\hline \multirow{3}{*}{ Compound } & \multirow{3}{*}{$\begin{array}{l}\text { Concentration } \\
\qquad(\mu \mathrm{g})\end{array}$} & \multicolumn{4}{|c|}{ Zone of inhibition (mm) } \\
\hline & & \multicolumn{2}{|c|}{ Gram-positive bacteria } & \multicolumn{2}{|c|}{ Gram-negative bacteria } \\
\hline & & S. aureus & B. subtilis & E. coli & K. pneumoniae \\
\hline \multirow[t]{2}{*}{$10 \mathrm{a}$} & 100 & 20 & 22 & 17 & 19 \\
\hline & 200 & 27 & 25 & 20 & 22 \\
\hline \multirow[t]{2}{*}{$10 \mathrm{~b}$} & 100 & 15 & 18 & 16 & 19 \\
\hline & 200 & 17 & 19 & 20 & 21 \\
\hline \multirow[t]{2}{*}{$10 \mathrm{c}$} & 100 & 25 & 22 & 19 & 23 \\
\hline & 200 & 30 & 25 & 22 & 28 \\
\hline \multirow[t]{2}{*}{$11 \mathrm{a}$} & 100 & 12 & 11 & 14 & 13 \\
\hline & 200 & 15 & 13 & 17 & 16 \\
\hline \multirow[t]{2}{*}{$11 b$} & 100 & 11 & 12 & 11 & 11 \\
\hline & 200 & 14 & 16 & 14 & 13 \\
\hline \multirow[t]{2}{*}{$11 \mathrm{c}$} & 100 & 14 & 14 & 10 & 11 \\
\hline & 200 & 17 & 18 & 12 & 13 \\
\hline \multirow[t]{2}{*}{$12 \mathrm{a}$} & 100 & 28 & 31 & 22 & 21 \\
\hline & 200 & 33 & 35 & 26 & 25 \\
\hline \multirow[t]{2}{*}{$12 b$} & 100 & 21 & 22 & 20 & 21 \\
\hline & 200 & 26 & 27 & 23 & 24 \\
\hline \multirow[t]{2}{*}{$12 \mathrm{c}$} & 100 & 32 & 34 & 23 & 26 \\
\hline & 200 & 35 & 28 & 25 & 27 \\
\hline \multirow[t]{2}{*}{ Chloramphenicol } & 100 & 35 & 38 & 40 & 42 \\
\hline & 200 & 39 & 41 & 44 & 45 \\
\hline
\end{tabular}

${ }^{1} \mathrm{H}-\mathrm{NMR}$ spectrum of $\mathbf{8 a}$ displayed broad signals in the regions 10.49 and $5.15 \mathrm{ppm}$ for $\mathrm{NH}$ and $\mathrm{NH}_{2}$ which disappeared on deuteration in addition to signals due to other pro- tons. The potassium dithiocarbazate of acid hydrazide (9) is prepared from 8 on treatment with carbon disulfide in the presence of potassium hydroxide under ultrasonic conditions. 
This on refluxion in acetic acid cyclized to $5^{\prime}$-(3-aroyl-4,5dihydro- $1 H$-pyrazole-4-sulfonylmethyl)- $\left[1^{\prime}, 3^{\prime}, 4^{\prime}\right]$ thiadiazole$2^{\prime}$-thiol (10). Acid catalysed hydrolysis of 9 resulted in 5'-(3-aroyl-4,5-dihydro-1 $H$-pyrazole-4-sulfonylmethyl)$\left[1^{\prime}, 3^{\prime}, 4^{\prime}\right]$ oxadiazole-2' - thiol (11). Further, the compound 9 on treatment with hydrazine hydrate produced $4^{\prime}$-amino5'-(3-aroyl-4,5-dihydro-1 $H$-pyrazole-4-sulfonylmethyl)$\left[1^{\prime}, 2^{\prime}, 4^{\prime}\right]$ triazole-3'-thiol (12) (Chart 2). The ${ }^{1} \mathrm{H}-\mathrm{NMR}$ spectra of 10-12 displayed a singlet in the region 10.24$10.35 \mathrm{ppm}$ for $\mathrm{SH}$ besides signals due to pyrazoline ring and methylene protons. In addition to these, 12a showed a broad singlet at $5.64 \mathrm{ppm}$ for $\mathrm{NH}_{2}$ which disappeared on deuteration. The structures of 5-7 and 10-12 are further confirmed by ${ }^{13} \mathrm{C}$-NMR spectra.

Antimicrobial Testing The compounds 10-12 were tested for in vitro antimicrobial activity at two different concentrations 100 and $200 \mu \mathrm{g}$ per disc. The antibacterial activity was screened against Staphylococcus aureus, Bacillus subtilis (Gram-positive bacteria) and Escherichia coli, Klebsiella pneumoniae (Gram-negative bacteria) on nutrient agar plates at $37^{\circ} \mathrm{C}$ for $24 \mathrm{~h}$ using chloramphenicol as reference drug. The compounds were also evaluated for their antifungal activity against Fusarium solani, Curvularia lunata and Aspergillus niger using ketoconazole as standard drug. Fungi cultures were grown on potato dextrose agar medium (PDA) at $25^{\circ} \mathrm{C}$ for $3 \mathrm{~d}$. The spore suspension was adjusted to $10^{6}$

Table 2. The in Vitro Antifungal Activity of $\mathbf{1 0 - 1 2}$

\begin{tabular}{|c|c|c|c|c|}
\hline \multirow{2}{*}{ Compound } & \multirow{2}{*}{$\begin{array}{l}\text { Concentration } \\
\qquad(\mu \mathrm{g})\end{array}$} & \multicolumn{3}{|c|}{ Zone of inhibition (mm) } \\
\hline & & F. solani & C. lunata & A. niger \\
\hline \multirow[t]{2}{*}{$10 \mathbf{a}$} & 100 & 24 & 28 & 26 \\
\hline & 200 & 27 & 30 & 31 \\
\hline \multirow[t]{2}{*}{$10 \mathrm{~b}$} & 100 & 23 & 24 & 22 \\
\hline & 200 & 27 & 25 & 26 \\
\hline \multirow[t]{2}{*}{$10 \mathrm{c}$} & 100 & 26 & 25 & 26 \\
\hline & 200 & 30 & 27 & 29 \\
\hline \multirow[t]{2}{*}{$11 \mathrm{a}$} & 100 & 19 & 15 & 17 \\
\hline & 200 & 20 & 22 & 21 \\
\hline \multirow[t]{2}{*}{$11 b$} & 100 & 15 & 14 & 18 \\
\hline & 200 & 20 & 18 & 23 \\
\hline \multirow[t]{2}{*}{$11 \mathrm{c}$} & 100 & 17 & 18 & 14 \\
\hline & 200 & 22 & 22 & 17 \\
\hline \multirow[t]{2}{*}{$12 \mathbf{a}$} & 100 & 33 & 34 & 31 \\
\hline & 200 & 35 & 36 & 37 \\
\hline \multirow[t]{2}{*}{$12 b$} & 100 & 27 & 28 & 32 \\
\hline & 200 & 32 & 34 & 35 \\
\hline \multirow[t]{2}{*}{$12 \mathrm{c}$} & 100 & 36 & 38 & 34 \\
\hline & 200 & 39 & 42 & 36 \\
\hline \multirow[t]{2}{*}{ Ketoconazole } & 100 & 38 & 41 & 36 \\
\hline & 200 & 42 & 44 & 39 \\
\hline
\end{tabular}

pores $\mathrm{ml}^{-1}$ at a $\mathrm{mg} \mathrm{ml}^{-1}$ concentration by the Vincent and Vincent method. ${ }^{17)}$

The results of the compounds of preliminary antimicrobial testing are shown in Tables 1 and 2. The results revealed that the inhibitory activity against Gram-positive bacteria was higher than Gram-negative bacteria. The oxadiazole derivatives 11a - c were displayed least activity. The compounds 10a, 10c, 12a and 12c showed excellent activity against Gram-positive bacteria (inhibitory zone $>25 \mathrm{~mm}$ ) and good activity against Gram-negative bacteria (inhibitory zone $>20 \mathrm{~mm}$ ). All the test compounds showed moderate to high inhibitory effect towards tested fungi. The presence of chloro substituent at position of the arylsulfonyl moiety enhances the antimicrobial activity.

The minimal inhibitory concentration (MIC) values were determined as the lowest concentration that completely inhibited visible growth of the microorganisms (Table 3 ). The structure-antimicrobial activity relationship of the synthesized compounds revealed that the compounds having pyrazoline with oxadiazole exhibited least activity when compared with compounds having pyrazoline with thiadiazole and triazole moieties. Among the substituents on the aryl group, 4-chlorophenyl derivatives were the most activity. The maximum activity was observed with the compounds 10c, 12a and 12c.

\section{Conclusion}

A new class of sulfone linked bis heterocycles, 5' (3-aroyl-4,5-dihydro-1 $H$-pyrazole-4-sulfonylmethyl)$\left[1^{\prime}, 3^{\prime}, 4^{\prime}\right]$ thiadiazole-2' -thiol (10), 5' -(3-aroyl-4,5-dihydro$1 H$-pyrazole-4-sulfonylmethyl)- $\left[1^{\prime}, 3^{\prime}, 4^{\prime}\right]$ oxadiazole- $2^{\prime}$-thiol (11) and 4' $4^{\prime}$-amino-5'-(3-aroyl-4,5-dihydro-1H-pyrazole-4-

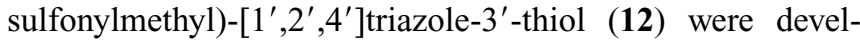
oped by the regiospecific 1,3-dipolar cycloaddition of diazomethane to aroylethenesulfonylacetic acid methyl ester (4) followed by functionalization of ester moiety with appropriate reagents. The antimibcrobial testing showed that the compounds having pyrazoline with thiadiazole and triazole units possess greater antibacterial activity.

\section{Experimental}

Melting points were determined in open capillaries on a Mel-Temp apparatus and are uncorrected. The purity of the compounds was checked by TLC (silica gel H, BDH, hexane-ethyl acetate, $3: 1$ ). The IR spectra were recorded on a Thermo Nicolet IR 200 FT-IR spectrometer as $\mathrm{KBr}$ pellets and the wave numbers were given in $\mathrm{cm}^{-1}$. The ${ }^{1} \mathrm{H}$-NMR spectra were recorded in $\mathrm{CDCl}_{3} / \mathrm{DMSO}-d_{6}$ on a Varian EM-360 spectrometer $(300 \mathrm{MHz})$. The ${ }^{13} \mathrm{C}-$ NMR spectra were recorded in $\mathrm{CDCl}_{3} / \mathrm{DMSO}-d_{6}$ on a Varian VXR spectrometer operating at $75.5 \mathrm{MHz}$. All chemical shifts are reported in $\delta(\mathrm{ppm})$ using TMS as an internal standard. The microanalyses were performed on Perkin-Elmer 240C elemental analyzer. The mass spectra were recorded on Finnigan Mat $1210 \mathrm{~B}$ and Waters Micromass Quattro Micro API at $70 \mathrm{eV}$ with an emission current of $100 \mu \mathrm{A}$. The starting compound 1-aroyl-2-

Table 3. Minimal Inhibitory Concentration of 10c, 12a and 12c

\begin{tabular}{|c|c|c|c|c|c|c|c|}
\hline \multirow{2}{*}{ Compound } & \multicolumn{7}{|c|}{ Minimal inhibitory concentration (MIC), $\mu \mathrm{g} / \mathrm{ml}$} \\
\hline & S. aureus & B. subtilis & E. coli & K. pneumoniae & F. solani & C. lunata & A. niger \\
\hline $10 \mathrm{c}$ & 100 & 200 & 200 & 200 & 100 & 100 & 200 \\
\hline $12 a$ & 50 & 100 & 100 & 100 & 100 & 100 & 100 \\
\hline $12 \mathrm{c}$ & 25 & 50 & 50 & 50 & 50 & 25 & 25 \\
\hline Chloramphenicol & 6.25 & 6.25 & 6.25 & 12.5 & - & - & - \\
\hline Ketoconazole & - & - & - & - & 12.5 & 6.25 & 6.25 \\
\hline
\end{tabular}


chloroethene (1) was prepared according to literature procedure. ${ }^{18)}$

General Procedure of Synthesis of Aroylethenemercapto Acetic Acid $(\mathbf{2 a}-\mathbf{c})$ To a solution of sodium hydroxide $(0.002 \mathrm{~mol})$ in methanol $(10 \mathrm{ml})$, mercaptoacetic acid $(0.001 \mathrm{~mol})$ was added dropwise. To this, compound $1(0.001 \mathrm{~mol})$ was added in portions and the reaction mixture was stirred at $0{ }^{\circ} \mathrm{C}$ for $3-4 \mathrm{~h}$. The contents were poured onto crushed ice and neutralized with dil. $\mathrm{HCl}$. The reaction mixture was extracted with ethyl acetate. The solvent when removed under reduced pressure gave the compound 2.

Benzoylethenemercapto Acetic Acid (2a): White solid, yield 71\%, mp $34-36{ }^{\circ} \mathrm{C}$; IR $(\mathrm{KBr}) \mathrm{cm}^{-1}$ : $3200(\mathrm{OH}), 1725(\mathrm{C}=\mathrm{O}), 1585(\mathrm{C}=\mathrm{C}) .{ }^{1} \mathrm{H}-$ NMR $\left(\mathrm{CDCl}_{3}\right) \delta: 3.74\left(\mathrm{~s}, 2 \mathrm{H}, \mathrm{CH}_{2}\right), 7.68\left(\mathrm{~d}, 1 \mathrm{H}, \mathrm{H}_{\mathrm{B}}, J=13.7 \mathrm{~Hz}\right), 7.93(\mathrm{~d}$ $\left.1 \mathrm{H}, \mathrm{H}_{\mathrm{A}}, J=13.7 \mathrm{~Hz}\right), 7.46-7.84(\mathrm{~m}, 5 \mathrm{H}, \mathrm{Ar}-\mathrm{H}), 9.91$ (br s, $\left.1 \mathrm{H}, \mathrm{OH}\right) ;{ }^{13} \mathrm{C}-$ NMR $\left(\mathrm{CDCl}_{3}\right) \delta: 53.4\left(\mathrm{CH}_{2}\right), 137.8(\mathrm{COCH}), 143.8(\mathrm{CHS}), 171.9(\mathrm{COOH})$, 184.1 (ArCO), 128.7, 131.1, 132.6, 135.5.

4-Methylbenzoylethenemercaptoacetic Acid (2b): White solid, yield 76\%, mp 45-47 ${ }^{\circ} \mathrm{C}$; IR $(\mathrm{KBr}) \mathrm{cm}^{-1}: 3214(\mathrm{OH}), 1722(\mathrm{C}=\mathrm{O}), 1581(\mathrm{C}=\mathrm{C}) .{ }^{1} \mathrm{H}-$ NMR $\left(\mathrm{CDCl}_{3}\right) \delta: 2.27\left(\mathrm{~s}, 3 \mathrm{H}, \mathrm{Ar}-\mathrm{CH}_{3}\right), 3.70\left(\mathrm{~s}, 2 \mathrm{H}, \mathrm{CH}_{2}\right), 7.71\left(\mathrm{~d}, 1 \mathrm{H}, \mathrm{H}_{\mathrm{B}}\right.$, $J=13.9 \mathrm{~Hz}), 7.95\left(\mathrm{~d}, 1 \mathrm{H}, \mathrm{H}_{\mathrm{A}}, J=13.9 \mathrm{~Hz}\right), 7.38-7.82(\mathrm{~m}, 4 \mathrm{H}, \mathrm{Ar}-\mathrm{H}), 10.1$ (brs, $1 \mathrm{H}, \mathrm{OH}) ;{ }^{13} \mathrm{C}-\mathrm{NMR}\left(\mathrm{CDCl}_{3}\right) \delta: 23.2\left(\mathrm{Ar}_{-} \mathrm{CH}_{3}\right), 53.8\left(\mathrm{CH}_{2}\right), 137.2$ $(\mathrm{COCH}), 143.3$ (CHS), $170.5(\mathrm{COOH}), 183.5$ (ArCO), 127.6, 130.4, 133.1, 139.5 .

4-Chlorobenzoylethenemercaptoacetic Acid (2c): White solid, yield 74\%, mp 67-69 ${ }^{\circ} \mathrm{C}$; IR $(\mathrm{KBr}) \mathrm{cm}^{-1}: 3218(\mathrm{OH}), 1714(\mathrm{C}=\mathrm{O}), 1574(\mathrm{C}=\mathrm{C}) .{ }^{1} \mathrm{H}-$ $\operatorname{NMR}\left(\mathrm{CDCl}_{3}\right) \delta: 3.79\left(\mathrm{~s}, 2 \mathrm{H}, \mathrm{CH}_{2}\right), 7.74\left(\mathrm{~d}, 1 \mathrm{H}, \mathrm{H}_{\mathrm{B}}, J=13.5 \mathrm{~Hz}\right), 7.98(\mathrm{~d}$ $\left.1 \mathrm{H}, \mathrm{H}_{\mathrm{A}}, J=13.5 \mathrm{~Hz}\right), 7.54-7.89(\mathrm{~m}, 4 \mathrm{H}, \mathrm{Ar}-\mathrm{H}), 9.82$ (br s, $\left.1 \mathrm{H}, \mathrm{OH}\right) ;{ }^{13} \mathrm{C}-$ NMR $\left(\mathrm{CDCl}_{3}\right) \delta: 54.1\left(\mathrm{CH}_{2}\right), 138.1(\mathrm{COCH}), 144.2$ (CHS), $171.4(\mathrm{COOH})$, 184.2 (ArCO), 128.4, 130.6, 132.4, 141.5.

General Procedure of Synthesis of Aroylethenesulfonylacetic Acid (3a-c) The compound $2(0.001 \mathrm{~mol})$ was subjected to oxidation with $30 \%$ hydrogen peroxide $(2 \mathrm{ml})$ in glacial acetic acid $(6.5 \mathrm{ml})$. The contents were stirred at $0{ }^{\circ} \mathrm{C}$ for $4 \mathrm{~h}$ and kept aside for $36 \mathrm{~h}$. The reaction mixture was poured onto crushed ice. The solid separated was filtered, dried and recrystallized from water.

Benzoylethenesulfonylacetic Acid (3a): White solid, 88\% yield, mp 132 $134{ }^{\circ} \mathrm{C}$; IR $(\mathrm{KBr}) \mathrm{cm}^{-1}: 3221(\mathrm{OH}), 1727(\mathrm{C}=\mathrm{O}), 1584(\mathrm{C}=\mathrm{C}), 1130,1326$ $\left(\mathrm{SO}_{2}\right) .{ }^{1} \mathrm{H}-\mathrm{NMR}\left(\mathrm{CDCl}_{3}\right) \delta: 4.38\left(\mathrm{~s}, 2 \mathrm{H}, \mathrm{CH}_{2}\right), 7.66\left(\mathrm{~d}, 1 \mathrm{H}, \mathrm{H}_{\mathrm{B}}, J=14.2 \mathrm{~Hz}\right)$, $7.96\left(\mathrm{~d}, 1 \mathrm{H}, \mathrm{H}_{\mathrm{A}}, J=14.2 \mathrm{~Hz}\right), 7.56-7.88(\mathrm{~m}, 5 \mathrm{H}, \mathrm{Ar}-\mathrm{H}), 9.68$ (br s, $1 \mathrm{H}$, $\mathrm{OH}) ;{ }^{13} \mathrm{C}-\mathrm{NMR}\left(\mathrm{CDCl}_{3}\right) \delta: 56.8\left(\mathrm{CH}_{2}\right), 138.7(\mathrm{COCH}), 141.8\left(\mathrm{CHSO}_{2}\right)$, 172.8 (COOH), 182.4 (ArCO), 128.4, 129.4, 132.6, 134.4.

4-Methylbenzoylethenesulfonylacetic Acid (3b): White solid, yield 85\%, mp $125-127^{\circ} \mathrm{C}$; IR $(\mathrm{KBr}) \mathrm{cm}^{-1}: 3224(\mathrm{OH}), 1714(\mathrm{C}=\mathrm{O}), 1568(\mathrm{C}=\mathrm{C})$, 1132, $1314\left(\mathrm{SO}_{2}\right) .{ }^{1} \mathrm{H}-\mathrm{NMR}\left(\mathrm{CDCl}_{3}\right) \delta: 2.24\left(\mathrm{~s}, 3 \mathrm{H}, \mathrm{Ar}-\mathrm{CH}_{3}\right), 4.40(\mathrm{~s}, 2 \mathrm{H}$, $\left.\mathrm{CH}_{2}\right), 7.63\left(\mathrm{~d}, 1 \mathrm{H}, \mathrm{H}_{\mathrm{B}}, J=14.4 \mathrm{~Hz}\right), 7.92\left(\mathrm{~d}, 1 \mathrm{H}, \mathrm{H}_{\mathrm{A}}, J=14.4 \mathrm{~Hz}\right), 7.42$ $7.80(\mathrm{~m}, 4 \mathrm{H}, \mathrm{Ar}-\mathrm{H}), 9.92(\mathrm{brs}, 1 \mathrm{H}, \mathrm{OH}) ;{ }^{13} \mathrm{C}-\mathrm{NMR}\left(\mathrm{CDCl}_{3}\right) \delta: 24.2(\mathrm{Ar}-$ $\left.\mathrm{CH}_{3}\right), 57.2\left(\mathrm{CH}_{2}\right), 137.6(\mathrm{COCH}), 142.3\left(\mathrm{CHSO}_{2}\right), 172.3(\mathrm{COOH}), 181.3$ (ArCO), 128.2, 129.2, 131.9, 135.4 .

4-Chlorobenzoylethenesulfonylacetic Acid (3c): White solid, yield 87\%, mp 151-153 ${ }^{\circ} \mathrm{C}$; IR $(\mathrm{KBr}) \mathrm{cm}^{-1}: 3218(\mathrm{OH}), 1724(\mathrm{C}=\mathrm{O}), 1564(\mathrm{C}=\mathrm{C})$, $1138,1336\left(\mathrm{SO}_{2}\right) .{ }^{1} \mathrm{H}-\mathrm{NMR}\left(\mathrm{CDCl}_{3}\right) \delta: 4.42\left(\mathrm{~s}, 2 \mathrm{H}, \mathrm{CH}_{2}\right), 7.69\left(\mathrm{~d}, 1 \mathrm{H}, \mathrm{H}_{\mathrm{B}}\right.$, $J=14.6 \mathrm{~Hz}), 7.98\left(\mathrm{~d}, 1 \mathrm{H}, \mathrm{H}_{\mathrm{A}}, J=14.6 \mathrm{~Hz}\right), 7.52-7.87(\mathrm{~m}, 4 \mathrm{H}, \mathrm{Ar}-\mathrm{H}), 9.96$ (brs, $1 \mathrm{H}, \mathrm{OH}) ;{ }^{13} \mathrm{C}-\mathrm{NMR}\left(\mathrm{CDCl}_{3}\right) \delta: 56.5\left(\mathrm{CH}_{2}\right), 138.1(\mathrm{COCH}), 142.6$ $\left(\mathrm{CHSO}_{2}\right), 173.2(\mathrm{COOH}), 179.6$ (ArCO), 128.1, 129.6, 132.3, 134.2.

General Procedure of Synthesis of Aroylethenesulfonylacetic Acid Methyl Ester $(4 \mathbf{a}-\mathbf{c})$ A solution of compound $3(0.001 \mathrm{~mol})$ in methanol $(10 \mathrm{ml})$ and sulfuric acid $(2 \mathrm{ml})$ was refluxed for $5-7 \mathrm{~h}$. The contents were cooled and poured onto crushed ice. The solid separated was filtered, dried and recrystallized from methanol.

Benzoylethenesulfonylacetic Acid Methyl Ester (4a): White crystals, yield, $85 \%, \mathrm{mp} 94-96^{\circ} \mathrm{C}$; IR $(\mathrm{KBr}) \mathrm{cm}^{-1}: 1746\left(\mathrm{CO}_{2} \mathrm{Me}\right), 1668(\mathrm{C}=\mathrm{O})$, $1579(\mathrm{C}=\mathrm{C}), 1142,1316\left(\mathrm{SO}_{2}\right) .{ }^{1} \mathrm{H}-\mathrm{NMR}\left(\mathrm{CDCl}_{3}\right) \delta: 3.78\left(\mathrm{~s}, 3 \mathrm{H}, \mathrm{OCH}_{3}\right)$, $4.36\left(\mathrm{~s}, 2 \mathrm{H}, \mathrm{CH}_{2}\right), 7.65\left(\mathrm{~d}, 1 \mathrm{H}, \mathrm{H}_{\mathrm{B}}, J=13.9 \mathrm{~Hz}\right), 7.91\left(\mathrm{~d}, 1 \mathrm{H}, \mathrm{H}_{\mathrm{A}}\right.$, $J=13.9 \mathrm{~Hz}), 7.36-7.52(\mathrm{~m}, 5 \mathrm{H}, \mathrm{Ar}-\mathrm{H}) ;{ }^{13} \mathrm{C}-\mathrm{NMR}\left(\mathrm{CDCl}_{3}\right) \delta: 53.1\left(\mathrm{OCH}_{3}\right)$, $59.6\left(\mathrm{CH}_{2}\right), 138.4(\mathrm{COCH}), 145.6\left(\mathrm{CHSO}_{2}\right), 168.1\left(\mathrm{CO}_{2} \mathrm{CH}_{3}\right), 182.4$ (ArCO), 130.5, 132.3, 137.5, 139.4. Anal. Calcd for $\mathrm{C}_{12} \mathrm{H}_{12} \mathrm{O}_{5} \mathrm{~S}: \mathrm{C}, 53.72 ; \mathrm{H}$, 4.51; Found: C, 53.78; H 4.49 .

4-Methylbenzoylethenesulfonylacetic Acid Methyl Ester (4b): White crystals, yield $89 \%$, mp $101-103{ }^{\circ} \mathrm{C}$; IR $(\mathrm{KBr}) \mathrm{cm}^{-1}: 1748\left(\mathrm{CO}_{2} \mathrm{Me}\right), 1662$ $(\mathrm{C}=\mathrm{O}), 1574(\mathrm{C}=\mathrm{C}), 1128,1341\left(\mathrm{SO}_{2}\right) .{ }^{1} \mathrm{H}-\mathrm{NMR}\left(\mathrm{CDCl}_{3}\right) \delta: 2.28(\mathrm{~s}, 3 \mathrm{H}$, $\left.\mathrm{Ar}-\mathrm{CH}_{3}\right), 3.72\left(\mathrm{~s}, 3 \mathrm{H}, \mathrm{OCH}_{3}\right), 4.32\left(\mathrm{~s}, 2 \mathrm{H}, \mathrm{CH}_{2}\right), 7.62\left(\mathrm{~d}, 1 \mathrm{H}, \mathrm{H}_{\mathrm{B}}\right.$, $J=13.7 \mathrm{~Hz}), 7.88\left(\mathrm{~d}, 1 \mathrm{H}, \mathrm{H}_{\mathrm{A}}, J=13.7 \mathrm{~Hz}\right), 7.32-7.54(\mathrm{~m}, 4 \mathrm{H}, \mathrm{Ar}-\mathrm{H}) ;{ }^{13} \mathrm{C}-$ NMR $\left(\mathrm{CDCl}_{3}\right) \delta: 23.1\left(\mathrm{Ar}_{-} \mathrm{CH}_{3}\right), 53.4\left(\mathrm{OCH}_{3}\right), 58.1\left(\mathrm{CH}_{2}\right), 138.1(\mathrm{COCH})$, $146.1\left(\mathrm{CHSO}_{2}\right), 167.4\left(\mathrm{CO}_{2} \mathrm{CH}_{3}\right), 181.3$ (ArCO), 129.4, 131.6, 136.3, 138.4. Anal. Calcd for $\mathrm{C}_{13} \mathrm{H}_{14} \mathrm{O}_{5} \mathrm{~S}$ : C, 55.31; H, 5.00; Found: C, 55.34; H 5.02.
4-Chlorobenzoylethenesulfonylacetic Acid Methyl Ester (4c): White crystals, yield $87 \%$, mp $124-126^{\circ} \mathrm{C}$; IR $(\mathrm{KBr}) \mathrm{cm}^{-1}$ : $1752\left(\mathrm{CO}_{2} \mathrm{Me}\right), 1669$ $(\mathrm{C}=\mathrm{O}), 1568(\mathrm{C}=\mathrm{C}), 1149,1325\left(\mathrm{SO}_{2}\right) .{ }^{1} \mathrm{H}-\mathrm{NMR}\left(\mathrm{CDCl}_{3}\right) \delta: 3.75(\mathrm{~s}, 3 \mathrm{H}$, $\left.\mathrm{OCH}_{3}\right), 4.38\left(\mathrm{~s}, 2 \mathrm{H}, \mathrm{CH}_{2}\right), 7.69\left(\mathrm{~d}, 1 \mathrm{H}, \mathrm{H}_{\mathrm{B}}, J=14.2 \mathrm{~Hz}\right), 7.94\left(\mathrm{~d}, 1 \mathrm{H}, \mathrm{H}_{\mathrm{A}}\right.$, $J=14.2 \mathrm{~Hz}), 7.48-7.72(\mathrm{~m}, 4 \mathrm{H}, \mathrm{Ar}-\mathrm{H}) ;{ }^{13} \mathrm{C}-\mathrm{NMR}\left(\mathrm{CDCl}_{3}\right) \delta: 53.8\left(\mathrm{OCH}_{3}\right)$, $58.9\left(\mathrm{CH}_{2}\right), 138.9(\mathrm{COCH}), 147.3\left(\mathrm{CHSO}_{2}\right), 168.6\left(\mathrm{CO}_{2} \mathrm{CH}_{3}\right), 181.6$ (ArCO), 128.9, 131.4, 135.8, 137.4. Anal. Calcd for $\mathrm{C}_{12} \mathrm{H}_{11} \mathrm{ClO}_{5} \mathrm{~S}$ : C, 47.61; H, 3.66; Found: C, 47.55; H, 3.69.

General Procedure of Synthesis of 1,1-Dioxo-6-phenacyl-[1,4,5]thiadiazinan-3-one $(5 \mathrm{a}-\mathrm{c})$ and (3-Aryl-4,5-dihydro-1 $H$-pyrazole-5-sulfonyl)acetic Acid Methyl Ester (6a-c) A mixture of $4(0.0025 \mathrm{~mol})$, hydrazine hydrate $(0.005 \mathrm{~mol})$ and methanol $(15 \mathrm{ml})$ was refluxed for $8-10 \mathrm{~h}$ and cooled. The solid separated was filtered and dried. It was purified by column chromatography (hexane-ethyl acetate, $3: 1$ ).

1,1-Dioxo-6-phenacyl-[1,4,5]thiadiazinan-3-one (5a): White crystals, yield $26 \%$, mp $102-104{ }^{\circ} \mathrm{C}$; IR $(\mathrm{KBr}) \mathrm{cm}^{-1}$ : $3284(\mathrm{NH}), 1682(\mathrm{C}=\mathrm{O})$, 1652 (NHCO), 1132, $1334\left(\mathrm{SO}_{2}\right) .{ }^{1} \mathrm{H}-\mathrm{NMR}\left(\mathrm{DMSO}-d_{6}\right) \delta: 2.82$ (br s, $1 \mathrm{H}$, $\mathrm{NH}), 3.50\left(\mathrm{~d}, 2 \mathrm{H}, \mathrm{COCH}_{2}\right), 4.28(\mathrm{~s}, 2 \mathrm{H}, \mathrm{H}-2), 4.57-4.59$ (m, 1H, H-6), $7.38-7.84(\mathrm{~m}, 5 \mathrm{H}, \mathrm{Ar}-\mathrm{H}), 8.50(\mathrm{~s}, 1 \mathrm{H}, \mathrm{CONH}) ;{ }^{13} \mathrm{C}-\mathrm{NMR}\left(\mathrm{DMSO}-d_{6}\right) \delta$ : $36.2\left(\mathrm{CH}_{2}\right), 59.4(\mathrm{C}-2), 72.8(\mathrm{C}-6), 172.6(\mathrm{CONH}), 198.6$ (ArCO), 127.7, 130.2, 133.1, 136.5; MS: $m / z=268\left(\mathrm{M}^{+*}\right)$. Anal. Calcd for $\mathrm{C}_{11} \mathrm{H}_{12} \mathrm{~N}_{2} \mathrm{O}_{4} \mathrm{~S}: \mathrm{C}$, 49.24; H, 4.51; N, 10.44; Found: C, 49.20; H, 4.55; N, 10.55.

1,1-Dioxo-6-(4-methylphenacyl)-[1,4,5]thiadiazinan-3-one $\mathbf{( 5 b ) : ~ W h i t e ~}$ crystals, yield $16 \%$, mp 97-99 ${ }^{\circ} \mathrm{C}$; IR $(\mathrm{KBr}) \mathrm{cm}^{-1}$ : $3286(\mathrm{NH}), 1678$ $(\mathrm{C}=\mathrm{O}), 1654$ (NHCO), 1130, $1332\left(\mathrm{SO}_{2}\right) .{ }^{1} \mathrm{H}-\mathrm{NMR}$ (DMSO- $\left.d_{6}\right) \delta: 2.25(\mathrm{~s}$, $\left.3 \mathrm{H}, \mathrm{Ar}-\mathrm{CH}_{3}\right), 2.78$ (br s, 1H, NH), $3.48\left(\mathrm{~d}, 2 \mathrm{H}, \mathrm{COCH}_{2}\right), 4.27$ (s, 2H, H-2), $4.54-4.57$ (m, 1H, H-6), 7.36-7.85 (m, 4H, Ar-H), 8.47 (s, 1H, CONH);

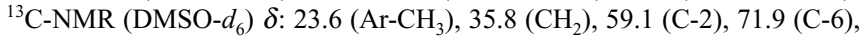
$169.3(\mathrm{CONH}), 198.3(\mathrm{C}=\mathrm{O}), 128.4,130.5,134.2,136.8$. Anal. Calcd for $\mathrm{C}_{12} \mathrm{H}_{14} \mathrm{~N}_{2} \mathrm{O}_{4} \mathrm{~S}$ : C, 51.05; H, 5.00; N, 9.92; Found: C, 51.16; H, 5.06; N, 9.99.

1,1-Dioxo-6-(4-chlorophenacyl)-[1,4,5]thiadiazinan-3-one $(\mathbf{5 c})$ : White crystals, yield $24 \%$, mp $135-137^{\circ} \mathrm{C}$; IR (KBr) cm $\mathrm{cm}^{-1}: 3286(\mathrm{NH}), 1684$ $(\mathrm{C}=\mathrm{O}), 1656(\mathrm{NHCO}), 1136,1338\left(\mathrm{SO}_{2}\right) .{ }^{1} \mathrm{H}-\mathrm{NMR}$ (DMSO- $\left.d_{6}\right) \delta: 2.82$ (br s, $1 \mathrm{H}, \mathrm{NH}), 3.53\left(\mathrm{~d}, 2 \mathrm{H}, \mathrm{COCH}_{2}\right), 4.31(\mathrm{~s}, 2 \mathrm{H}, \mathrm{H}-2), 4.55-4.58(\mathrm{~m}, 1 \mathrm{H}$, $\mathrm{H}-6), 7.44-7.91(\mathrm{~m}, 4 \mathrm{H}, \mathrm{Ar}-\mathrm{H}), 8.44(\mathrm{~s}, 1 \mathrm{H}, \mathrm{CONH}) ;{ }^{13} \mathrm{C}-\mathrm{NMR}$ (DMSO$\left.d_{6}\right) \delta: 37.1\left(\mathrm{CH}_{2}\right), 58.5(\mathrm{C}-2), 72.6(\mathrm{C}-6), 171.8(\mathrm{CONH}), 197.8(\mathrm{C}=\mathrm{O})$, 128.2, 131.4, 133.7, 135.9. Anal. Calcd for $\mathrm{C}_{11} \mathrm{H}_{11} \mathrm{ClN}_{2} \mathrm{O}_{4} \mathrm{~S}: \mathrm{C}, 43.64 ; \mathrm{H}$, 3.66; N, 9.25; Found: C, 43.72; H, 3.59; N, 9.32 .

(3-Phenyl-4,5-dihydro-1 $H$-pyrazole-5-sulfonyl)-acetic Acid Methyl Ester (6a): Yellow solid, yield 62\%, mp 142-144 ${ }^{\circ} \mathrm{C}$; IR $(\mathrm{KBr}) \mathrm{cm}^{-1}: 3290(\mathrm{NH})$, $1742(\mathrm{C}=\mathrm{O}), 1558(\mathrm{C}=\mathrm{N}), 1125,1336\left(\mathrm{SO}_{2}\right) .{ }^{1} \mathrm{H}-\mathrm{NMR}\left(\mathrm{CDCl}_{3}\right) \delta: 3.53(\mathrm{~d}$, $2 \mathrm{H}, \mathrm{H}-4), 3.72\left(\mathrm{~s}, 3 \mathrm{H}, \mathrm{OCH}_{3}\right), 4.39\left(\mathrm{~s}, 2 \mathrm{H}, \mathrm{SO}_{2} \mathrm{CH}_{2}\right), 4.62-4.65(\mathrm{~m}, 1 \mathrm{H}, \mathrm{H}-$ 5), $7.20-7.69(\mathrm{~m}, 5 \mathrm{H}, \mathrm{Ar}-\mathrm{H}), 10.22(\mathrm{brs}, 1 \mathrm{H}, \mathrm{NH}) ;{ }^{13} \mathrm{C}-\mathrm{NMR}\left(\mathrm{CDCl}_{3}\right) \delta$ : $32.8(\mathrm{C}-4), 47.5\left(\mathrm{SO}_{2} \mathrm{CH}_{2}\right), 52.2\left(\mathrm{OCH}_{3}\right), 67.2(\mathrm{C}-5), 152.2(\mathrm{C}-3), 161.8$ $(\mathrm{C}=\mathrm{O}), 129.5,130.4,131.5,133.2$; MS: $m / z=282\left(\mathrm{M}^{+*}\right)$. Anal. Calcd for $\mathrm{C}_{12} \mathrm{H}_{14} \mathrm{~N}_{2} \mathrm{O}_{4} \mathrm{~S}$ : C, 51.05; H, 5.00; N, 9.92; Found: C, 51.02; H, 5.06; N, 9.86 . (3-(4-Methylphenyl)-4,5-dihydro- $1 H$-pyrazole-5-sulfonyl)-acetic Acid Methyl Ester (6b): Yellow solid, yield $69 \%$, mp $158-160{ }^{\circ} \mathrm{C}$; IR $(\mathrm{KBr})$ $\mathrm{cm}^{-1}: 3288(\mathrm{NH}), 1748(\mathrm{C}=\mathrm{O}), 1552(\mathrm{C}=\mathrm{N}), 1122,1343\left(\mathrm{SO}_{2}\right) .{ }^{1} \mathrm{H}-\mathrm{NMR}$ $\left(\mathrm{CDCl}_{3}\right) \delta: 2.32\left(\mathrm{~s}, 3 \mathrm{H}, \mathrm{Ar}-\mathrm{CH}_{3}\right), 3.49(\mathrm{~d}, 2 \mathrm{H}, \mathrm{H}-4), 3.70\left(\mathrm{~s}, 3 \mathrm{H}, \mathrm{OCH}_{3}\right)$, $4.34\left(\mathrm{~s}, 2 \mathrm{H}, \mathrm{SO}_{2} \mathrm{CH}_{2}\right), 4.60-4.64(\mathrm{~m}, 1 \mathrm{H}, \mathrm{H}-5), 7.19-7.74(\mathrm{~m}, 4 \mathrm{H}, \mathrm{Ar}-\mathrm{H})$,

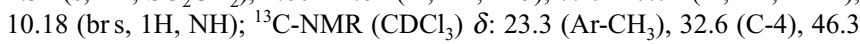
$\left(\mathrm{SO}_{2} \mathrm{CH}_{2}\right), 51.8\left(\mathrm{OCH}_{3}\right), 67.1(\mathrm{C}-5), 151.9(\mathrm{C}-3), 162.5(\mathrm{C}=\mathrm{O}), 129.2$, 130.8, 131.5, 132.6. Anal. Calcd for $\mathrm{C}_{13} \mathrm{H}_{16} \mathrm{~N}_{2} \mathrm{O}_{4} \mathrm{~S}: \mathrm{C}, 52.69 ; \mathrm{H}, 5.44 ; \mathrm{N}$, 9.45; Found: C, 52.74; H, 5.51; N, 9.51 .

(3-(4-Chlorophenyl)-4,5-dihydro-1H-pyrazole-5-sulfonyl)-acetic Acid Methyl Ester (6c): Yellow solid, yield $65 \%, \mathrm{mp} 149-151^{\circ} \mathrm{C}$; IR $(\mathrm{KBr})$ $\mathrm{cm}^{-1}: 3310(\mathrm{NH}), 1755(\mathrm{C}=\mathrm{O}), 1554(\mathrm{C}=\mathrm{N}), 1140,1335\left(\mathrm{SO}_{2}\right) .{ }^{1} \mathrm{H}-\mathrm{NMR}$ $\left(\mathrm{CDCl}_{3}\right) \delta: 3.57(\mathrm{~d}, 2 \mathrm{H}, \mathrm{H}-4), 3.75\left(\mathrm{~s}, 3 \mathrm{H}, \mathrm{OCH}_{3}\right), 4.38\left(\mathrm{~s}, 2 \mathrm{H}, \mathrm{SO}_{2} \mathrm{CH}_{2}\right)$, $4.65-4.68(\mathrm{~m}, 1 \mathrm{H}, \mathrm{H}-5), 7.25-7.78(\mathrm{~m}, 4 \mathrm{H}, \mathrm{Ar}-\mathrm{H}), 10.25$ (br s, $1 \mathrm{H}, \mathrm{NH})$; ${ }^{13} \mathrm{C}-\mathrm{NMR}\left(\mathrm{CDCl}_{3}\right) \delta: 32.9(\mathrm{C}-4), 47.9\left(\mathrm{SO}_{2} \mathrm{CH}_{2}\right), 52.7\left(\mathrm{OCH}_{3}\right), 67.5(\mathrm{C}-5)$, $151.9(\mathrm{C}-3), 162.1(\mathrm{C}=\mathrm{O}), 129.2,130.3,131.6,132.3$. Anal. Calcd for $\mathrm{C}_{12} \mathrm{H}_{13} \mathrm{ClN}_{2} \mathrm{O}_{4} \mathrm{~S}: \mathrm{C}, 45.50 ; \mathrm{H}, 4.14 ; \mathrm{N}, 8.84$; Found: C, 45.59; H, 4.20; N, 8.89 .

General Procedure of Synthesis of (3-Aroyl-4,5-dihydro-1 H-pyrazole4-sulfonyl)-acetic Acid Methyl Ester $(7 \mathbf{a}-\mathbf{c})$ To a cooled solution of 4 $(0.005 \mathrm{~mol})$ in dichloromethane $(20 \mathrm{ml})$, an ethereal solution of diazomethane $(40 \mathrm{ml}, 0.4 \mathrm{M})$ and triethylamine $(0.12 \mathrm{~g})$ were added. The reaction mixture was kept at -20 to $-15^{\circ} \mathrm{C}$ for $40-48 \mathrm{~h}$. The solvent was removed under reduced pressure. The resultant solid was purified by column chromatography (hexane-ethyl acetate, $4: 1$ ).

(3-Benzoyl-4,5-dihydro-1H-pyrazole-4-sulfonyl)-acetic Acid Methyl Ester (7a): Yellow solid, yield 75\%, mp 136-138 ${ }^{\circ} \mathrm{C}$; IR $(\mathrm{KBr}) \mathrm{cm}^{-1}: 3348$ $(\mathrm{NH}), 1746(\mathrm{C}=\mathrm{O}), 1684$ (ArCO), $1576(\mathrm{C}=\mathrm{N}), 1125,1328\left(\mathrm{SO}_{2}\right) .{ }^{1} \mathrm{H}-$ 
NMR $\left(\mathrm{CDCl}_{3}\right) \delta: 3.76\left(\mathrm{~s}, 3 \mathrm{H}, \mathrm{OCH}_{3}\right), 3.94\left(\mathrm{dd}, 1 \mathrm{H}, \mathrm{H}_{\mathrm{X}}, J_{\mathrm{AX}}=5.4 \mathrm{~Hz}\right.$, $\left.J_{\mathrm{MX}}=10.3 \mathrm{~Hz}\right), 4.32\left(\mathrm{~s}, 2 \mathrm{H}, \mathrm{SO}_{2} \mathrm{CH}_{2}\right), 4.53\left(\mathrm{dd}, 1 \mathrm{H}, \mathrm{H}_{\mathrm{M}}, J_{\mathrm{MX}}=10.3 \mathrm{~Hz}\right.$, $\left.J_{\mathrm{AM}}=12.6 \mathrm{~Hz}\right), 5.07\left(\mathrm{dd}, 1 \mathrm{H}, \mathrm{H}_{\mathrm{A}}, J_{\mathrm{AX}}=5.4 \mathrm{~Hz}, J_{\mathrm{AM}}=12.6 \mathrm{~Hz}\right), 7.23-7.72$ (m, 5H, Ar-H), 10.52 (br s, $1 \mathrm{H}, \mathrm{NH}) ;{ }^{13} \mathrm{C}-\mathrm{NMR}\left(\mathrm{CDCl}_{3}\right) \delta: 48.3\left(\mathrm{SO}_{2} \mathrm{CH}_{2}\right)$, $52.4\left(\mathrm{OCH}_{3}\right), 57.0(\mathrm{C}-5), 65.8(\mathrm{C}-4), 152.2(\mathrm{C}-3), 162.4\left(\mathrm{CO}_{2} \mathrm{CH}_{3}\right), 185.6$ (ArCO), 128.7, 129.8, 131.5, 132.4. MS: $m / z=310\left(\mathrm{M}^{+}\right)$. Anal. Calcd for $\mathrm{C}_{13} \mathrm{H}_{14} \mathrm{~N}_{2} \mathrm{O}_{5} \mathrm{~S}$ : C, 50.31; H, 4.55; N, 9.03; Found: C, 50.38; H, 4.50; N, 9.13.

(3-(4-Methylbenzoyl)-4,5-dihydro- $1 H$-pyrazole-4-sulfonyl)-acetic Acid Methyl Ester (7b): Yellow solid, yield $71 \%$, mp $155-157^{\circ} \mathrm{C}$; IR $(\mathrm{KBr})$ $\mathrm{cm}^{-1}: 3343(\mathrm{NH}), 1743(\mathrm{C}=\mathrm{O}), 1678(\mathrm{ArCO}), 1569(\mathrm{C}=\mathrm{N}), 1132,1323$ $\left(\mathrm{SO}_{2}\right) .{ }^{1} \mathrm{H}-\mathrm{NMR}\left(\mathrm{CDCl}_{3}\right) \delta: 2.23\left(\mathrm{~s}, 3 \mathrm{H}, \mathrm{Ar}_{-} \mathrm{CH}_{3}\right), 3.72\left(\mathrm{~s}, 3 \mathrm{H}, \mathrm{OCH}_{3}\right), 3.87$ $\left(\mathrm{dd}, 1 \mathrm{H}, \mathrm{H}_{\mathrm{X}}, J_{\mathrm{AX}}=5.2 \mathrm{~Hz}, J_{\mathrm{MX}}=10.1 \mathrm{~Hz}\right), 4.36\left(\mathrm{~s}, 2 \mathrm{H}, \mathrm{SO}_{2} \mathrm{CH}_{2}\right), 4.56(\mathrm{dd}$, $\left.1 \mathrm{H}, \mathrm{H}_{\mathrm{M}}, J_{\mathrm{MX}}=10.1 \mathrm{~Hz}, J_{\mathrm{AM}}=12.1 \mathrm{~Hz}\right), 5.00\left(\mathrm{dd}, 1 \mathrm{H}, \mathrm{H}_{\mathrm{A}}, J_{\mathrm{AX}}=5.2 \mathrm{~Hz}\right.$, $\left.J_{\mathrm{AM}}=12.1 \mathrm{~Hz}\right), 7.16-7.70(\mathrm{~m}, 4 \mathrm{H}, \mathrm{Ar}-\mathrm{H}), 10.46(\mathrm{br} \mathrm{s}, 1 \mathrm{H}, \mathrm{NH}) ;{ }^{13} \mathrm{C}-\mathrm{NMR}$ $\left(\mathrm{CDCl}_{3}\right) \delta: 24.5\left(\mathrm{CH}_{3}\right), 47.9\left(\mathrm{SO}_{2} \mathrm{CH}_{2}\right), 52.2\left(\mathrm{OCH}_{3}\right), 56.7(\mathrm{C}-5), 66.2(\mathrm{C}-4)$ 153.2 (C-3), $161.9\left(\mathrm{CO}_{2} \mathrm{CH}_{3}\right), 186.1$ (ArCO), 127.8, 128.9, 129.4, 131.6. Anal. Calcd for $\mathrm{C}_{14} \mathrm{H}_{16} \mathrm{~N}_{2} \mathrm{O}_{5} \mathrm{~S}$ : C, 51.84; H, 4.97; N, 8.64; Found: C, 51.76; $\mathrm{H}, 4.94 ; \mathrm{N}, 8.55$.

(3-(4-Chlorobenzoyl)-4,5-dihydro-1H-pyrazole-4-sulfonyl)-acetic Acid Methyl Ester (7c): Yellow solid, yield $74 \%$, mp $163-165^{\circ} \mathrm{C}$; IR $(\mathrm{KBr})$ $\mathrm{cm}^{-1}: 3335(\mathrm{NH}), 1749(\mathrm{C}=\mathrm{O}), 1686(\mathrm{ArCO}), 1582(\mathrm{CN}), 1120,1332$ $\left(\mathrm{SO}_{2}\right) .{ }^{1} \mathrm{H}-\mathrm{NMR}\left(\mathrm{CDCl}_{3}\right) \delta: 3.78\left(\mathrm{~s}, 3 \mathrm{H}, \mathrm{OCH}_{3}\right), 3.90\left(\mathrm{dd}, 1 \mathrm{H}, \mathrm{H}_{\mathrm{X}}\right.$, $\left.J_{\mathrm{AX}}=5.6 \mathrm{~Hz}, J_{\mathrm{MX}}=10.6 \mathrm{~Hz}\right), 4.34\left(\mathrm{~s}, 2 \mathrm{H}, \mathrm{SO}_{2} \mathrm{CH}_{2}\right), 4.54\left(\mathrm{dd}, 1 \mathrm{H}, \mathrm{H}_{\mathrm{M}}\right.$, $\left.J_{\mathrm{MX}}=10.6 \mathrm{~Hz}, J_{\mathrm{AM}}=12.9 \mathrm{~Hz}\right), 5.10\left(\mathrm{dd}, 1 \mathrm{H}, \mathrm{H}_{\mathrm{A}}, J_{\mathrm{AX}}=5.6 \mathrm{~Hz}, J_{\mathrm{AM}}=12.9 \mathrm{~Hz}\right)$ $7.23-7.76(\mathrm{~m}, 4 \mathrm{H}, \mathrm{Ar}-\mathrm{H}), 10.55$ (br s, $1 \mathrm{H}, \mathrm{NH}) ;{ }^{13} \mathrm{C}-\mathrm{NMR}\left(\mathrm{CDCl}_{3}\right) \delta: 48.7$ $\left(\mathrm{SO}_{2} \mathrm{CH}_{2}\right), 52.6\left(\mathrm{OCH}_{3}\right), 57.0(\mathrm{C}-5), 65.7$ (C-4), 152.3 (C-3), 162.8 $\left(\mathrm{CO}_{2} \mathrm{CH}_{3}\right), 185.9$ (ArCO), 128.7, 129.6, 131.9, 132.7. Anal. Calcd for $\mathrm{C}_{13} \mathrm{H}_{13} \mathrm{ClN}_{2} \mathrm{O}_{5} \mathrm{~S}$ : C, 45.29; H, 3.80; N, 8.13; Found: C, 45.23; H, 3.84; N, 8.20 .

General Procedure of Synthesis of (3-Aroyl-4,5-dihydro- $1 \mathrm{H}$-pyrazole4-sulfonyl)-acetic Acid Hydrazide (8a-c) To a solution of $7(0.001 \mathrm{~mol})$ in absolute ethanol $(5 \mathrm{ml})$, hydrazine hydrate $(0.0045 \mathrm{~mol})$ and pyridine $(0.4 \mathrm{ml})$ was added and stirred for $4-5 \mathrm{~h}$ at room temperature. The resultant solid was filtered, dried and recrystallized from ethanol.

(3-Benzoyl-4,5-dihydro-1 $H$-pyrazole-4-sulfonyl)-acetic Acid Hydrazide (8a): Yellow solid, yield $75 \%, \mathrm{mp} 149-151{ }^{\circ} \mathrm{C}$; IR $(\mathrm{KBr}) \mathrm{cm}^{-1}: 3320(\mathrm{NH})$, $3225\left(\mathrm{NH}_{2}\right), 1662(\mathrm{C}=\mathrm{O}), 1653(\mathrm{ArCO}), 1578(\mathrm{C}=\mathrm{N}), 1135,1320\left(\mathrm{SO}_{2}\right)$. ${ }^{1} \mathrm{H}-\mathrm{NMR}\left(\mathrm{CDCl}_{3}\right) \delta: 3.89\left(\mathrm{dd}, 1 \mathrm{H}, \mathrm{H}_{\mathrm{X}}, J_{\mathrm{AX}}=5.3 \mathrm{~Hz}, J_{\mathrm{MX}}=10.5 \mathrm{~Hz}\right), 4.30(\mathrm{~s}$, $\left.2 \mathrm{H}, \mathrm{SO}_{2} \mathrm{CH}_{2}\right), 4.42\left(\mathrm{dd}, 1 \mathrm{H}, \mathrm{H}_{\mathrm{M}}, J_{\mathrm{MX}}=10.5 \mathrm{~Hz}, J_{\mathrm{AM}}=12.5 \mathrm{~Hz}\right), 5.00(\mathrm{dd}, 1 \mathrm{H}$, $\left.\mathrm{H}_{\mathrm{A}}, J_{\mathrm{AX}}=5.3 \mathrm{~Hz}, J_{\mathrm{AM}}=12.5 \mathrm{~Hz}\right), 5.15\left(\right.$ br s, $\left.2 \mathrm{H}, \mathrm{NH}_{2}\right), 7.21-7.70(\mathrm{~m}, 5 \mathrm{H}$, $\mathrm{Ar}-\mathrm{H}), 9.49$ (br s, $1 \mathrm{H}, \mathrm{CONH}), 10.49$ (br s, $1 \mathrm{H}, \mathrm{NH}) ;{ }^{13} \mathrm{C}-\mathrm{NMR}\left(\mathrm{CDCl}_{3}\right) \delta$ : 48.8 (C-5), $57.4\left(\mathrm{SO}_{2} \mathrm{CH}_{2}\right), 65.7(\mathrm{C}-4), 152.6(\mathrm{C}-3), 170.9(\mathrm{CONH}), 185.9$ (ArCO), 128.1, 128.9, 130.6, 132.0.

(3-(4-Methylbenzoyl)-4,5-dihydro- $1 H$-pyrazole-4-sulfonyl)-acetic Acid Hydrazide (8b): Yellow solid, yield $80 \%, \mathrm{mp} 166-168^{\circ} \mathrm{C}$; IR $(\mathrm{KBr}) \mathrm{cm}^{-1}$. $3316(\mathrm{NH}), 3223\left(\mathrm{NH}_{2}\right), 1665(\mathrm{C}=\mathrm{O}), 1655(\mathrm{ArCO}), 1575(\mathrm{C}=\mathrm{N}), 1140$ $1334\left(\mathrm{SO}_{2}\right) .{ }^{1} \mathrm{H}-\mathrm{NMR}\left(\mathrm{CDCl}_{3}\right) \delta: 2.25\left(\mathrm{~s}, 3 \mathrm{H}, \mathrm{Ar}^{-\mathrm{CH}_{3}}\right), 3.93\left(\mathrm{dd}, 1 \mathrm{H}, \mathrm{H}_{\mathrm{X}}\right.$, $\left.J_{\mathrm{AX}}=5.1 \mathrm{~Hz}, J_{\mathrm{MX}}=10.3 \mathrm{~Hz}\right), 4.33\left(\mathrm{~s}, 2 \mathrm{H}, \mathrm{SO}_{2} \mathrm{CH}_{2}\right), 4.42\left(\mathrm{dd}, 1 \mathrm{H}, \mathrm{H}_{\mathrm{M}}\right.$ $\left.J_{\mathrm{MX}}=10.3 \mathrm{~Hz}, J_{\mathrm{AM}}=12.3 \mathrm{~Hz}\right), 4.96\left(\mathrm{dd}, 1 \mathrm{H}, \mathrm{H}_{\mathrm{A}}, J_{\mathrm{AX}}=5.1 \mathrm{~Hz}, J_{\mathrm{AM}}=12.3 \mathrm{~Hz}\right)$ 5.12 (brs, $\left.2 \mathrm{H}, \mathrm{NH}_{2}\right), 7.19-7.73(\mathrm{~m}, 4 \mathrm{H}, \mathrm{Ar}-\mathrm{H}), 9.45$ (br s, $\left.1 \mathrm{H}, \mathrm{CONH}\right)$ 10.51 (br s, $1 \mathrm{H}, \mathrm{NH}) ;{ }^{13} \mathrm{C}-\mathrm{NMR}\left(\mathrm{CDCl}_{3}\right) \delta: 24.2\left(\mathrm{Ar}^{\left.-\mathrm{CH}_{3}\right)}, 48.4(\mathrm{C}-5), 57.1\right.$ $\left(\mathrm{SO}_{2} \mathrm{CH}_{2}\right), 64.9$ (C-4), 151.3 (C-3), $169.8(\mathrm{CONH}), 185.4$ (ArCO), 127.3, $128.2,130.2,131.9$.

(3-(4-Chlorobenzoyl)-4,5-dihydro-1 $H$-pyrazole-4-sulfonyl)-acetic Acid Hydrazide (8c): Yellow solid, yield $78 \%, \mathrm{mp} 178-180^{\circ} \mathrm{C}$; IR $(\mathrm{KBr}) \mathrm{cm}^{-1}$ : $3322(\mathrm{NH}), 3228\left(\mathrm{NH}_{2}\right), 1669(\mathrm{C}=\mathrm{O}), 1658(\mathrm{ArCO}), 1580(\mathrm{C}=\mathrm{N}), 1138$, $1340\left(\mathrm{SO}_{2}\right) .{ }^{1} \mathrm{H}-\mathrm{NMR}\left(\mathrm{CDCl}_{3}\right) \quad \delta: 4.01\left(\mathrm{dd}, 1 \mathrm{H}, \mathrm{H}_{\mathrm{X}}, J_{\mathrm{AX}}=5.5 \mathrm{~Hz}\right.$, $\left.J_{\mathrm{MX}}=10.8 \mathrm{~Hz}\right), 4.35\left(\mathrm{~s}, 2 \mathrm{H}, \mathrm{SO}_{2} \mathrm{CH}_{2}\right), 4.49\left(\mathrm{dd}, 1 \mathrm{H}, \mathrm{H}_{\mathrm{M}}, J_{\mathrm{MX}}=10.8 \mathrm{~Hz}\right.$, $\left.J_{\mathrm{AM}}=12.7 \mathrm{~Hz}\right), 5.07\left(\mathrm{dd}, 1 \mathrm{H}, \mathrm{H}_{\mathrm{A}}, J_{\mathrm{AX}}=5.5 \mathrm{~Hz}, J_{\mathrm{AM}}=12.7 \mathrm{~Hz}\right), 5.17(\mathrm{br} \mathrm{s}, 2 \mathrm{H}$ $\mathrm{NH}_{2}$ ), $7.25-8.14$ (m, 4H, Ar-H), 9.38 (br s, $1 \mathrm{H}, \mathrm{CONH}$ ), 10.54 (br s, $1 \mathrm{H}$, $\mathrm{NH}) ;{ }^{13} \mathrm{C}$-NMR $\left(\mathrm{CDCl}_{3}\right) \delta: 49.2(\mathrm{C}-5), 57.7\left(\mathrm{SO}_{2} \mathrm{CH}_{2}\right), 65.7(\mathrm{C}-4), 152.3$ (C-3), $171.3(\mathrm{CONH}), 186.3$ (ArCO), 128.6, 129.5, 130.4, 132.3.

General Procedure of Synthesis of Potassium (3-Aroyl-4,5-dihydro$1 H$-pyrazole-4-sulfonylacetyl)-hydrazine- $N^{\prime}$-carbodithioate $(9 a-c)$ To a mixture of potassium hydroxide $(0.002 \mathrm{~mol})$ and compound $8(0.001 \mathrm{~mol})$ in absolute ethanol $(5 \mathrm{ml})$, carbon disulfide $(0.004 \mathrm{~mol})$ was added and sonicated for $10-12 \mathrm{~h}$. The separated solid was filtered and dried.

General Procedure of Synthesis of 5'-(3-Aroyl-4,5-dihydro-1H-pyrazole-4-sulfonylmethyl)-[1', $\left.\mathbf{3}^{\prime}, \mathbf{4}^{\prime}\right]$ thiadiazole-2' -thiol $(\mathbf{1 0 a}-\mathbf{c})$ The compound $9(0.001 \mathrm{~mol})$ in acetic acid $(4 \mathrm{ml})$ was refluxed for $20-24 \mathrm{~h}$. It was cooled and poured onto crushed ice. The solid obtained was filtered, dried and recrystallized from 2-propanol.

5'-(3-Benzoyl-4,5-dihydro- $1 H$-pyrazole-4-sulfonylmethyl)-[1', $\left.3^{\prime}, 4^{\prime}\right]$ thiadiazole-2' -thiol (10a): Yellow solid, yield $71 \%$, mp $198-200^{\circ} \mathrm{C}$; IR $(\mathrm{KBr})$ $\mathrm{cm}^{-1}: 3324(\mathrm{NH}), 2558(\mathrm{SH}), 1568(\mathrm{C}=\mathrm{N}), 1146,1338\left(\mathrm{SO}_{2}\right) \cdot{ }^{1} \mathrm{H}-\mathrm{NMR}$ $\left(\right.$ DMSO$\left.d_{6}\right) \delta: 3.95\left(\mathrm{dd}, 1 \mathrm{H}, \mathrm{H}_{\mathrm{X}}, J_{\mathrm{AX}}=5.4 \mathrm{~Hz}, J_{\mathrm{MX}}=10.3 \mathrm{~Hz}\right), 4.31(\mathrm{~s}, 2 \mathrm{H}$, $\left.\mathrm{SO}_{2} \mathrm{CH}_{2}\right), 4.74\left(\mathrm{dd}, 1 \mathrm{H}, \mathrm{H}_{\mathrm{M}}, J_{\mathrm{MX}}=10.3 \mathrm{~Hz}, J_{\mathrm{AM}}=12.4 \mathrm{~Hz}\right), 5.15\left(\mathrm{dd}, 1 \mathrm{H}, \mathrm{H}_{\mathrm{A}}\right.$, $\left.J_{\mathrm{AX}}=5.4 \mathrm{~Hz}, J_{\mathrm{AM}}=12.4 \mathrm{~Hz}\right), 7.23-7.76(\mathrm{~m}, 5 \mathrm{H}, \mathrm{Ar}-\mathrm{H}), 10.27(\mathrm{~s}, 1 \mathrm{H}, \mathrm{SH})$, $10.46(\mathrm{br} \mathrm{s}, 1 \mathrm{H}, \mathrm{NH}) ;{ }^{13} \mathrm{C}-\mathrm{NMR}\left(\mathrm{DMSO}-d_{6}\right) \delta: 51.8(\mathrm{C}-5), 57.0\left(\mathrm{SO}_{2} \mathrm{CH}_{2}\right)$, 65.8 (C-4), 152.2 (C-3), $163.4\left(\mathrm{C}-2^{\prime}\right), 168.5\left(\mathrm{C}-5^{\prime}\right), 186.8(\mathrm{C}=\mathrm{O}), 127.9$, 128.6, 129.5, 131.4; MS $m / z: 368\left(\mathrm{M}^{+\cdot}\right)$. Anal. Calcd for $\mathrm{C}_{13} \mathrm{H}_{12} \mathrm{~N}_{4} \mathrm{O}_{3} \mathrm{~S}_{3}: \mathrm{C}$, 42.38; H, 3.28; N, 15.21; Found: C, 42.42; H, 3.24; N, 15.29 .

5'-(3-(4-Methylbenzoyl)-4,5-dihydro-1 $H$-pyrazole-4-sulfonylmethyl)$\left[1^{\prime}, 3^{\prime}, 4^{\prime}\right]$ thiadiazole-2'-thiol (10b): Yellow solid, yield $76 \%$, mp 222$224^{\circ} \mathrm{C}$; IR (KBr) cm ${ }^{-1}: 3328(\mathrm{NH}), 2555(\mathrm{SH}), 1564(\mathrm{C}=\mathrm{N}), 1140,1330$ $\left(\mathrm{SO}_{2}\right) .{ }^{1} \mathrm{H}-\mathrm{NMR}\left(\mathrm{DMSO}-d_{6}\right) \delta: 2.28\left(\mathrm{~s}, 3 \mathrm{H}, \mathrm{Ar}-\mathrm{CH}_{3}\right), 3.94\left(\mathrm{dd}, 1 \mathrm{H}, \mathrm{H}_{\mathrm{X}}\right.$, $\left.J_{\mathrm{AX}}=5.2 \mathrm{~Hz}, J_{\mathrm{MX}}=10.1 \mathrm{~Hz}\right), 4.39\left(\mathrm{~s}, 2 \mathrm{H}, \mathrm{SO}_{2} \mathrm{CH}_{2}\right), 4.59\left(\mathrm{dd}, 1 \mathrm{H}, \mathrm{H}_{\mathrm{M}}\right.$, $\left.J_{\mathrm{MX}}=10.1 \mathrm{~Hz}, J_{\mathrm{AM}}=12.2 \mathrm{~Hz}\right), 5.11\left(\mathrm{dd}, 1 \mathrm{H}, \mathrm{H}_{\mathrm{A}}, J_{\mathrm{AX}}=5.2 \mathrm{~Hz}, J_{\mathrm{AM}}=12.2 \mathrm{~Hz}\right)$, $7.18-7.69(\mathrm{~m}, 4 \mathrm{H}, \mathrm{Ar}-\mathrm{H}), 10.29(\mathrm{~s}, 1 \mathrm{H}, \mathrm{SH}), 10.52(\mathrm{br} \mathrm{s}, 1 \mathrm{H}, \mathrm{NH}) ;{ }^{13} \mathrm{C}-$ NMR (DMSO- $\left.d_{6}\right) \delta: 24.6\left(\mathrm{Ar}^{-\mathrm{CH}_{3}}\right), 51.1(\mathrm{C}-5), 56.4\left(\mathrm{SO}_{2} \mathrm{CH}_{2}\right), 65.6(\mathrm{C}-4)$, $151.7(\mathrm{C}-3), 166.9\left(\mathrm{C}-2^{\prime}\right), 167.8\left(\mathrm{C}-5^{\prime}\right), 186.4(\mathrm{C}=\mathrm{O}), 127.6,128.7,129.4$, 131.2; MS m/z: $382\left(\mathrm{M}^{+\cdot}\right)$. Anal. Calcd for $\mathrm{C}_{14} \mathrm{H}_{14} \mathrm{~N}_{4} \mathrm{O}_{3} \mathrm{~S}_{3}$ : C, 43.96; $\mathrm{H}$, 3.69 ; N, 14.65; Found: C, 43.89; H, 3.74; N; 14.58 .

5'-(3-(4-Chlorobenzoyl)-4,5-dihydro- $1 H$-pyrazole-4-sulfonylmethyl)$\left[1^{\prime}, 3^{\prime}, 4^{\prime}\right]$ thiadiazole-2'-thiol (10c): Yellow solid, yield 73\%, mp 237$239^{\circ} \mathrm{C}$; IR $(\mathrm{KBr}) \mathrm{cm}^{-1}: 3336(\mathrm{NH}), 2562(\mathrm{SH}), 1566(\mathrm{C}=\mathrm{N}), 1148,1342$ $\left(\mathrm{SO}_{2}\right) .{ }^{1} \mathrm{H}-\mathrm{NMR} \quad\left(\mathrm{DMSO}-d_{6}\right) \quad \delta: 3.91\left(\mathrm{dd}, 1 \mathrm{H}, \mathrm{H}_{\mathrm{X}}, \quad J_{\mathrm{AX}}=5.6 \mathrm{~Hz}\right.$, $\left.J_{\mathrm{MX}}=10.6 \mathrm{~Hz}\right), 4.34\left(\mathrm{~s}, 2 \mathrm{H}, \mathrm{SO}_{2} \mathrm{CH}_{2}\right), 4.55\left(\mathrm{dd}, 1 \mathrm{H}, \mathrm{H}_{\mathrm{M}}, J_{\mathrm{MX}}=10.6 \mathrm{~Hz}\right.$, $\left.J_{\mathrm{AM}}=12.6 \mathrm{~Hz}\right), 5.17\left(\mathrm{dd}, 1 \mathrm{H}, \mathrm{H}_{\mathrm{A}}, J_{\mathrm{AX}}=5.6 \mathrm{~Hz}, J_{\mathrm{AM}}=12.6 \mathrm{~Hz}\right), 7.25-7.69$ (m, 4H, Ar-H), 10.24 (s, 1H, SH), 10.57 (br s, $1 \mathrm{H}, \mathrm{NH}) ;{ }^{13} \mathrm{C}-\mathrm{NMR}$ (DMSO$\left.d_{6}\right) \delta: 52.3(\mathrm{C}-5), 57.6\left(\mathrm{SO}_{2} \mathrm{CH}_{2}\right), 66.5(\mathrm{C}-4), 152.8(\mathrm{C}-3), 166.5\left(\mathrm{C}-2^{\prime}\right)$, $168.5\left(\mathrm{C}-5^{\prime}\right), 187.6(\mathrm{C}=\mathrm{O}), 128.5,129.9,131.6,132.5$; $\mathrm{MS} m / z: 402\left(\mathrm{M}^{+}\right)$. Anal. Calcd for $\mathrm{C}_{13} \mathrm{H}_{11} \mathrm{ClN}_{4} \mathrm{O}_{3} \mathrm{~S}_{3}$ : C, 38.75; H, 2.75; N, 13.91; Found: C, 38.70; H, 2.77; N, 13.98.

General Procedure of Synthesis of 5'-(3-Aroyl-4,5-dihydro- $1 \mathrm{H}$-pyrazole-4-sulfonylmethyl)- $\left[1^{\prime}, 3^{\prime}, 4^{\prime}\right]$ oxadiazole-2'-thiol $(11 \mathrm{a}-\mathrm{c})$ The compound $9(0.001 \mathrm{~mol})$ was dissolved in $6 \mathrm{ml}$ of water and acidified with $1-$ $2 \mathrm{ml}$ of conc. $\mathrm{HCl}$. The regenerated solid was collected by filtration, dried and purified by recrystallization from 2-propanol.

5'-(3-Benzoyl-4,5-dihydro-1H-pyrazole-4-sulfonylmethyl)-[1', $\left.3^{\prime}, 4^{\prime}\right]$ oxadiazole-2' -thiol (11a): Yellow solid, yield $73 \%, \mathrm{mp} 169-171^{\circ} \mathrm{C}$; IR $(\mathrm{KBr})$ $\mathrm{cm}^{-1}: 3340(\mathrm{NH}), 2560(\mathrm{SH}), 1565(\mathrm{C}=\mathrm{N}), 1130,1335\left(\mathrm{SO}_{2}\right) .{ }^{1} \mathrm{H}-\mathrm{NMR}$ $\left(\right.$ DMSO- $\left.d_{6}\right) \delta: 3.88\left(\mathrm{dd}, 1 \mathrm{H}, \mathrm{H}_{\mathrm{X}}, J_{\mathrm{AX}}=5.5 \mathrm{~Hz}, J_{\mathrm{MX}}=10.4 \mathrm{~Hz}\right), 4.37(\mathrm{~s}, 2 \mathrm{H}$, $\left.\mathrm{SO}_{2} \mathrm{CH}_{2}\right), 4.69\left(\mathrm{dd}, 1 \mathrm{H}, \mathrm{H}_{\mathrm{M}}, J_{\mathrm{MX}}=10.4 \mathrm{~Hz}, J_{\mathrm{AM}}=12.5 \mathrm{~Hz}\right), 5.19\left(\mathrm{dd}, 1 \mathrm{H}, \mathrm{H}_{\mathrm{A}}\right.$, $\left.J_{\mathrm{AX}}=5.5 \mathrm{~Hz}, J_{\mathrm{AM}}=12.5 \mathrm{~Hz}\right), 7.26-7.73(\mathrm{~m}, 5 \mathrm{H}, \mathrm{Ar}-\mathrm{H}), 10.26(\mathrm{~s}, 1 \mathrm{H}, \mathrm{SH})$, 10.49 (br s, $1 \mathrm{H}, \mathrm{NH}) ;{ }^{13} \mathrm{C}-\mathrm{NMR}\left(\mathrm{DMSO}-d_{6}\right) \delta: 51.7(\mathrm{C}-5), 57.4\left(\mathrm{SO}_{2} \mathrm{CH}_{2}\right)$, $66.2(\mathrm{C}-4), 152.5(\mathrm{C}-3), 160.8\left(\mathrm{C}-2^{\prime}\right), 168.6\left(\mathrm{C}^{\prime} 5^{\prime}\right), 187.8(\mathrm{C}=\mathrm{O}), 127.5$, 128.6, 129.7, 131.8; MS $m / z: 352\left(\mathrm{M}^{+}\right)$. Anal. Calcd for $\mathrm{C}_{13} \mathrm{H}_{12} \mathrm{~N}_{4} \mathrm{O}_{4} \mathrm{~S}_{2}: \mathrm{C}$, 44.31 ; H, 3.43; N, 15.90; Found: C, 44.37; H, 3.46; N, 15.82 .

5'-(3-(4-Methylbenzoyl)-4,5-dihydro- $1 H$-pyrazole-4-sulfonylmethyl)$\left[1^{\prime}, 3^{\prime}, 4^{\prime}\right]$ oxadiazole- $2^{\prime}$-thiol (11b): Yellow solid, yield $68 \%$, mp 188 $190^{\circ} \mathrm{C}$; IR $(\mathrm{KBr}) \mathrm{cm}^{-1}: 3345(\mathrm{NH}), 2556(\mathrm{SH}), 1563(\mathrm{C}=\mathrm{N}), 1138,1337$ $\left(\mathrm{SO}_{2}\right) .{ }^{1} \mathrm{H}-\mathrm{NMR}\left(\mathrm{DMSO}-d_{6}\right) \delta: 2.25\left(\mathrm{~s}, 3 \mathrm{H}, \mathrm{Ar}-\mathrm{CH}_{3}\right), 3.86\left(\mathrm{dd}, 1 \mathrm{H}, \mathrm{H}_{\mathrm{X}}\right.$, $\left.J_{\mathrm{AX}}=5.2 \mathrm{~Hz}, J_{\mathrm{MX}}=10.2 \mathrm{~Hz}\right), 4.41\left(\mathrm{~s}, 2 \mathrm{H}, \mathrm{SO}_{2} \mathrm{CH}_{2}\right), 4.73\left(\mathrm{dd}, 1 \mathrm{H}, \mathrm{H}_{\mathrm{M}}\right.$, $\left.J_{\mathrm{MX}}=10.2 \mathrm{~Hz}, J_{\mathrm{AM}}=12.3 \mathrm{~Hz}\right), 5.13\left(\mathrm{dd}, 1 \mathrm{H}, \mathrm{H}_{\mathrm{A}}, J_{\mathrm{AX}}=5.2 \mathrm{~Hz}, J_{\mathrm{AM}}=12.3 \mathrm{~Hz}\right)$, $7.14-7.68(\mathrm{dd}, 4 \mathrm{H}, \mathrm{Ar}-\mathrm{H}), 10.28(\mathrm{~s}, 1 \mathrm{H}, \mathrm{SH}), 10.54$ (br s, $1 \mathrm{H}, \mathrm{NH}) ;{ }^{13} \mathrm{C}-$ NMR (DMSO- $\left.d_{6}\right) \delta: 25.2\left(\mathrm{Ar}^{-\mathrm{CH}_{3}}\right), 51.3(\mathrm{C}-5), 56.7\left(\mathrm{SO}_{2} \mathrm{CH}_{2}\right), 65.9(\mathrm{C}-4)$, $151.2(\mathrm{C}-3), 160.1\left(\mathrm{C}-2^{\prime}\right), 168.2\left(\mathrm{C}-5^{\prime}\right), 188.2(\mathrm{C}=\mathrm{O}), 127.8,129.5,132.4$, 133.5; MS m/z: $366\left(\mathrm{M}^{+}\right)$. Anal. Calcd for $\mathrm{C}_{14} \mathrm{H}_{14} \mathrm{~N}_{4} \mathrm{O}_{4} \mathrm{~S}_{2}: \mathrm{C}, 45.89 ; \mathrm{H}$, 3.85; N, 15.29; Found: C, 45.94; H, 3.87; N, 15.36.

5'-(3-(4-Chlorobenzoyl)-4,5-dihydro- $1 H$-pyrazole-4-sulfonylmethyl)$\left[1^{\prime}, 3^{\prime}, 4^{\prime}\right]$ oxadiazole-2'-thiol (11c): Yellow solid, yield $71 \%$, mp $197-$ $199^{\circ} \mathrm{C}$; IR ( KBr) cm ${ }^{-1}: 3349(\mathrm{NH}), 2564(\mathrm{SH}), 1567(\mathrm{C}=\mathrm{N}), 1127,1342$ $\left(\mathrm{SO}_{2}\right) .{ }^{1} \mathrm{H}-\mathrm{NMR}\left(\right.$ DMSO- $\left.d_{6}\right) \delta: 3.90\left(\mathrm{dd}, 1 \mathrm{H}, \mathrm{H}_{\mathrm{X}}, J_{\mathrm{AX}}=5.6 \mathrm{~Hz}, J_{\mathrm{MX}}=\right.$ $10.7 \mathrm{~Hz}), 4.46\left(\mathrm{~s}, 2 \mathrm{H}, \quad \mathrm{SO}_{2} \mathrm{CH}_{2}\right), 4.76\left(\mathrm{dd}, 1 \mathrm{H}, \mathrm{H}_{\mathrm{M}}, J_{\mathrm{MX}}=10.7 \mathrm{~Hz}\right.$, $\left.J_{\mathrm{AM}}=12.7 \mathrm{~Hz}\right), 5.12\left(\mathrm{dd}, 1 \mathrm{H}, \mathrm{H}_{\mathrm{A}}, J_{\mathrm{AX}}=5.6 \mathrm{~Hz}, J_{\mathrm{AM}}=12.7 \mathrm{~Hz}\right), 7.28-7.73$ (m, 4H, Ar-H), $10.32(\mathrm{~s}, 1 \mathrm{H}, \mathrm{SH}), 10.51$ (br s, $1 \mathrm{H}, \mathrm{NH}) ;{ }^{13} \mathrm{C}-\mathrm{NMR}$ (DMSO$\left.d_{6}\right) \delta: 52.1(\mathrm{C}-5), 57.6\left(\mathrm{SO}_{2} \mathrm{CH}_{2}\right), 66.5(\mathrm{C}-4), 152.8(\mathrm{C}-3), 161.2\left(\mathrm{C}-2^{\prime}\right)$, $168.8\left(\mathrm{C}-5^{\prime}\right), 187.5(\mathrm{C}=\mathrm{O}), 128.1,129.4,130.5,132.9$; $\mathrm{MS} m / z: 386\left(\mathrm{M}^{+*}\right)$. Anal. Calcd for $\mathrm{C}_{13} \mathrm{H}_{11} \mathrm{ClN}_{4} \mathrm{O}_{4} \mathrm{~S}_{2}$ : C, 40.36; H, 2.87; N, 14.48; Found: C, $40.39 ; \mathrm{H}, 2.85 ; \mathrm{N}, 14.40$.

General Procedure of Synthesis of $4^{\prime}$-Amino-5' -(3-aroyl-4,5-dihydro-

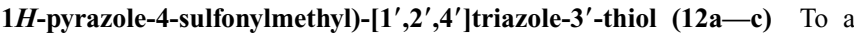
solution of $9(0.001 \mathrm{~mol})$ in $6 \mathrm{ml}$ of water, hydrazine hydrate $(0.002 \mathrm{~mol})$ was added and refluxed for $7-10 \mathrm{~h}$. The contents of the flask were cooled, diluted with water and acidified with $2 \mathrm{ml}$ of acetic acid. The separated solid was collected by filtration, dried and recrystallized from 2-propanol.

$4^{\prime}$-Amino-5' -(3-benzoyl-4,5-dihydro- $1 H$-pyrazole-4-sulfonylmethyl)- 
$\left[1^{\prime}, 2^{\prime}, 4^{\prime}\right]$ triazole-3' - thiol (12a): Yellow solid, yield $77 \%$, mp $210-212^{\circ} \mathrm{C}$; IR $(\mathrm{KBr}) \mathrm{cm}^{-1}: 3325(\mathrm{NH}), 3242\left(\mathrm{NH}_{2}\right), 2557(\mathrm{SH}), 1570(\mathrm{C}=\mathrm{N}), 1137$, $1334\left(\mathrm{SO}_{2}\right) .{ }^{1} \mathrm{H}-\mathrm{NMR}\left(\mathrm{DMSO}-d_{6}\right) \delta: 4.07\left(\mathrm{dd}, 1 \mathrm{H}, \mathrm{H}_{\mathrm{X}}, J_{\mathrm{AX}}=5.5 \mathrm{~Hz}\right.$, $\left.J_{\mathrm{MX}}=10.5 \mathrm{~Hz}\right), 4.36\left(\mathrm{~s}, 2 \mathrm{H}, \mathrm{SO}_{2} \mathrm{CH}_{2}\right), 4.77\left(\mathrm{dd}, 1 \mathrm{H}, \mathrm{H}_{\mathrm{M}}, J_{\mathrm{MX}}=10.5 \mathrm{~Hz}\right.$, $\left.J_{\mathrm{AM}}=12.7 \mathrm{~Hz}\right), 5.18\left(\mathrm{dd}, 1 \mathrm{H}, \mathrm{H}_{\mathrm{A}}, J_{\mathrm{AX}}=5.5 \mathrm{~Hz}, J_{\mathrm{AM}}=12.7 \mathrm{~Hz}\right), 5.64(\mathrm{br} \mathrm{s}, 2 \mathrm{H}$, $\mathrm{NH}_{2}$ ), $7.14-7.72$ (m, 5H, Ar-H), 10.29 (s, 1H, SH), 10.44 (br s, 1H, NH); ${ }^{13} \mathrm{C}-\mathrm{NMR}\left(\mathrm{DMSO}-d_{6}\right) \delta: 50.7(\mathrm{C}-5), 56.8\left(\mathrm{SO}_{2} \mathrm{CH}_{2}\right), 65.6(\mathrm{C}-4), 151.5(\mathrm{C}-$ 3), $153.4\left(\mathrm{C}-5^{\prime}\right), 155.6\left(\mathrm{C}-3^{\prime}\right), 188.8(\mathrm{C}=\mathrm{O}), 128.3,131.3,132.4,134.2$; MS m/z: $366\left(\mathrm{M}^{+\cdot}\right)$. Anal. Calcd for $\mathrm{C}_{13} \mathrm{H}_{14} \mathrm{~N}_{6} \mathrm{O}_{3} \mathrm{~S}_{2}: \mathrm{C}, 42.61 ; \mathrm{H}, 3.85 ; \mathrm{N}, 22.94$; Found: C, 42.56; H, 3.80; N, 23.05.

4'-Amino-5' -(3-(4-Methylbenzoyl)-4,5-dihydro-1 $H$-pyrazole-4-sulfonylmethyl)-[1' $\left., 2^{\prime}, 4^{\prime}\right]$ triazole-3' - thiol (12b): Yellow solid, yield 71\%, mp $202-$ $204^{\circ} \mathrm{C}$; IR $(\mathrm{KBr}) \mathrm{cm}^{-1}$ : $3337(\mathrm{NH}), 3245\left(\mathrm{NH}_{2}\right), 2548(\mathrm{SH}), 1573(\mathrm{C}=\mathrm{N})$, 1137, $1334\left(\mathrm{SO}_{2}\right) .{ }^{1} \mathrm{H}-\mathrm{NMR}\left(\mathrm{DMSO}-d_{6}\right) \delta: 2.27$ (s, 3H, Ar-CH $), 4.04$ (dd, $\left.1 \mathrm{H}, \mathrm{H}_{\mathrm{X}}, J_{\mathrm{AX}}=5.3 \mathrm{~Hz}, J_{\mathrm{MX}}=10.3 \mathrm{~Hz}\right), 4.37\left(\mathrm{~s}, 2 \mathrm{H}, \mathrm{SO}_{2} \mathrm{CH}_{2}\right), 4.68(\mathrm{dd}, 1 \mathrm{H}$, $\left.\mathrm{H}_{\mathrm{M}}, \quad J_{\mathrm{MX}}=10.3 \mathrm{~Hz}, J_{\mathrm{AM}}=12.5 \mathrm{~Hz}\right), 5.19\left(\mathrm{dd}, 1 \mathrm{H}, \mathrm{H}_{\mathrm{A}}, J_{\mathrm{AX}}=5.3 \mathrm{~Hz}\right.$, $\left.J_{\mathrm{AM}}=12.5 \mathrm{~Hz}\right), 5.61\left(\right.$ br s, $\left.2 \mathrm{H}, \mathrm{NH}_{2}\right), 7.09-7.68(\mathrm{~m}, 4 \mathrm{H}, \mathrm{Ar}-\mathrm{H}), 10.33(\mathrm{~s}$, $1 \mathrm{H}, \mathrm{SH}), 10.49$ (br s, $1 \mathrm{H}, \mathrm{NH}) ;{ }^{13} \mathrm{C}-\mathrm{NMR}\left(\mathrm{DMSO}-d_{6}\right) \delta: 23.5\left(\mathrm{Ar}-\mathrm{CH}_{3}\right)$, $50.3(\mathrm{C}-5), 55.4\left(\mathrm{SO}_{2} \mathrm{CH}_{2}\right), 65.9(\mathrm{C}-4), 150.2(\mathrm{C}-3), 142.3\left(\mathrm{C}-3^{\prime}\right), 153.1(\mathrm{C}-$ 5'), $187.6(\mathrm{C}=\mathrm{O}), 129.6,131.5,132.7,134.5$; MS m/z: $380\left(\mathrm{M}^{+\cdot}\right)$. Anal. Calcd for $\mathrm{C}_{14} \mathrm{H}_{16} \mathrm{~N}_{6} \mathrm{O}_{3} \mathrm{~S}_{2}$ : C, 44.20; H, 4.24; N, 22.09; Found: C, 44.25; H, 4.28; N, 21.98 .

4'-Amino-5'-(3-(4-chlorobenzoyl)-4,5-dihydro-1 $H$-pyrazole-4-sulfonylmethyl)-[1',2',4']triazole-3' -thiol (12c): Yellow solid, yield 74\%, mp 246 $248^{\circ} \mathrm{C}$; IR $(\mathrm{KBr}) \mathrm{cm}^{-1}$ : $3330(\mathrm{NH}), 3248\left(\mathrm{NH}_{2}\right), 2559(\mathrm{SH}), 1569(\mathrm{C}=\mathrm{N})$, 1144, $1345\left(\mathrm{SO}_{2}\right) .{ }^{1} \mathrm{H}-\mathrm{NMR}\left(\mathrm{DMSO}-d_{6}\right) \delta: 4.09\left(\mathrm{dd}, 1 \mathrm{H}, \mathrm{H}_{\mathrm{X}}, J_{\mathrm{AX}}=5.6 \mathrm{~Hz}\right.$, $\left.J_{\mathrm{MX}}=10.8 \mathrm{~Hz}\right), 4.40\left(\mathrm{~s}, 2 \mathrm{H}, \mathrm{SO}_{2} \mathrm{CH}_{2}\right), 4.78\left(\mathrm{dd}, 1 \mathrm{H}, \mathrm{H}_{\mathrm{M}}, J_{\mathrm{MX}}=10.8 \mathrm{~Hz}\right.$, $\left.J_{\mathrm{AM}}=12.8 \mathrm{~Hz}\right), 5.25\left(\mathrm{dd}, 1 \mathrm{H}, \mathrm{H}_{\mathrm{A}}, J_{\mathrm{AX}}=5.6 \mathrm{~Hz}, J_{\mathrm{AM}}=12.8 \mathrm{~Hz}\right), 5.67(\mathrm{br} \mathrm{s}, 2 \mathrm{H}$, $\mathrm{NH}_{2}$ ), $7.28-7.82(\mathrm{~m}, 4 \mathrm{H}, \mathrm{Ar}-\mathrm{H}), 10.35$ (s, 1H, SH), 10.43 (br s, 1H, NH); ${ }^{13} \mathrm{C}-\mathrm{NMR}\left(\mathrm{DMSO}-d_{6}\right) \delta: 50.9(\mathrm{C}-5), 57.1\left(\mathrm{SO}_{2} \mathrm{CH}_{2}\right), 65.8(\mathrm{C}-4), 151.9(\mathrm{C}-$ 3), $142.6\left(\mathrm{C}-3^{\prime}\right), 153.4\left(\mathrm{C}-5^{\prime}\right), 188.7(\mathrm{C}=\mathrm{O}), 129.2,131.7,132.4,133.6$; MS $m / z: 400\left(\mathrm{M}^{+*}\right)$. Anal. Calcd for $\mathrm{C}_{13} \mathrm{H}_{13} \mathrm{ClN}_{6} \mathrm{O}_{3} \mathrm{~S}_{2}: \mathrm{C}, 38.95 ; \mathrm{H}, 3.27 ; \mathrm{N}$, 20.96; Found: C, 38.96; H, 3.29; N, 20.89.

Antimicrobial Testing The compounds 10-12 were dissolved in DMSO at different concentrations of 100,200 and $800 \mu \mathrm{g} / \mathrm{ml}$.

Antibacterial and Antifungal Assays Preliminary antimicrobial activities of 10 - 12 compounds were tested by Agar disc-diffusion method. Sterile filter paper discs ( $6 \mathrm{~mm}$ diameter) moistened with the test compound solution in acetone of specific concentration $100 \mu \mathrm{g}$ and $200 \mu \mathrm{g} / \mathrm{disc}$ were carefully placed on the agar culture plates that had been previously inoculated separately with the microorganisms. The plates were incubated at $37^{\circ} \mathrm{C}$ and the diameter of the growth inhibition zones were measured after $24 \mathrm{~h}$ in case of bacteria and after $48 \mathrm{~h}$ in case of fungi.

The MICs of the compounds assays were carried out using microdilution susceptibility method. Chloramphenicol was used as reference antibacterial agent. Ketroconazole was used as reference antifungal agent. The test compounds, chloramphenicol and ketoconazole were dissolved in DMSO at concentration of $800 \mu \mathrm{g} / \mathrm{ml}$. The two-fold dilution of the solution was prepared
$(400,200,100, \ldots, 6.25 \mu \mathrm{g} / \mathrm{ml})$. The microorganism suspensions were inoculated to the corresponding wells. The plates were incubated at $36^{\circ} \mathrm{C}$ for 24 and $48 \mathrm{~h}$ for bacteria and fungi, respectively. The minimum inhibitory concentrations of the compounds were recorded as the lowest concentration of each chemical compounds in the tubes with no turbidity (i.e. no growth) of inoculated bacteria/fungi.

Acknowledgements The Department of Science and Technology (DST), New Delhi, India, is gratefully acknowledged for its financial support.

\section{References}

1) Holla B. S., Gonsalves R., Shenoy S., Eur. J. Med. Chem., 35, 267271 (2000).

2) Tinperciuc B., Parvu A., Palage M., Oniga O., Ghiran D., Farmacia (Bucharest), 47, 77-84 (1999).

3) Lutwick L. L., Rytel W. M., J. Am. Med. Assoc., 241, 271-272 (1979).

4) Fromtling R. A., Clin. Microbiol. Rev., 1, 187-208 (1988).

5) Godefroi E. F., Heeres J., Van Cutsem J., J. Med. Chem., 12, 784-791 (1969).

6) Odds F. C., Abbott A. B., J. Antimicrob. Chemother., 14, 105-114 (1984).

7) Shadomy S., White S. C., Yu H. P., J. Infect. Dis., 152, 1249-1256 (1985).

8) Demir-Erol D., Çalıs Ü., Demirdamar R., Yuluğ N., Ertan M., J. Pharm. Sci., 84, 462-465 (1995).

9) Katrizky A., "Comprehensive Heterocyclic Chemistry," Vol. 6, Pergamon, Oxford, 1984.

10) Diamond S., Sevrain C., WO 2003016870 (2003) [Chem. Abstr., 138, 198554 (2003)].

11) Nakao H., Matsuzaki Y., Tohnishi M., Mmorimoto M., Fujioka S., Takemoto T., Mamezuka K., WO 2002092584 (2002) [Chem. Abstr., 137, 384845 (2002)].

12) Vashi B. S., Mehta D. S., Shah V. H., Indian J. Chem., 35B, 111-115 (1996).

13) Kariyone K., Harada H., Kuritha M., Takaano T., J. Antibiot., 23, $131-136$ (1970).

14) Dannahardt G., Kiefer W., Kramer G., Maehrlein S., Nowe U., Fiebich B., Eur. J. Med. Chem., 35, 499-510 (2000).

15) Bhaskar Reddy D., Seshamma T., Venkata Ramana Reddy B., Acta Chimica Hungarica, 122, 19-24 (1986).

16) Padmavathi V., Rajagopal Sarma M., Venugopal Reddy K., Padmaja A., Reddy D. B., Heteroatom Chem., 14, 155-159 (2003).

17) Vincent J. C., Vincent H. W., Proc. Soc. Exp. Biol. Med., 55, 162-164 (1944).

18) Bhaskar Reddy D., Chandrasekhar Babu N., Padmavathi V., Sumathi R. P., Synthesis, 1999, 491-494 (1999). 